TRANSACTIONS OF THE

AMERICAN MATHEMATICAL SOCIETY

Volume 358, Number 3 , Pages 1317-1345

S 0002-9947(05)03840-7

Article electronically published on August 1, 2005

\title{
INVARIANT PRE-FOLIATIONS FOR NON-RESONANT NON-UNIFORMLY HYPERBOLIC SYSTEMS
}

\author{
ERNEST FONTICH, RAFAEL DE LA LLAVE, AND PAU MARTÍN
}

\begin{abstract}
Given an orbit whose linearization has invariant subspaces satisfying some non-resonance conditions in the exponential rates of growth, we prove existence of invariant manifolds tangent to these subspaces. The exponential rates of growth can be understood either in the sense of Lyapunov exponents or in the sense of exponential dichotomies. These manifolds can correspond to "slow manifolds", which characterize the asymptotic convergence.

Let $\left\{x_{i}\right\}_{i \in \mathbb{N}}$ be a regular orbit of a $C^{2}$ dynamical system $f$. Let $S$ be a subset of its Lyapunov exponents. Assume that all the Lyapunov exponents in $S$ are negative and that the sums of Lyapunov exponents in $S$ do not agree with any Lyapunov exponent in the complement of $S$. Denote by $E_{x_{i}}^{S}$ the linear spaces spanned by the spaces associated to the Lyapunov exponents in $S$. We show that there are smooth manifolds $W_{x_{i}}^{S}$ such that $f\left(W_{x_{i}}^{S}\right) \subset W_{x_{i+1}}^{S}$ and $T_{x_{i}} W_{x_{i}}^{S}=E_{x_{i}}^{S}$. We establish the same results for orbits satisfying dichotomies and whose rates of growth satisfy similar non-resonance conditions. These systems of invariant manifolds are not, in general, a foliation.
\end{abstract}

\section{INTRODUCTION AND STATEMENT OF RESULTS}

When studying the behavior of an orbit of a dynamical system $f$, it is natural to study the behavior of its linearization and wonder whether there are non-linear analogues for the features found in the study of the linearization.

Very often we can classify the tangent vectors along an orbit into subspaces with different rates of exponential growth either in the future or in the past. In the literature, there are several precise definitions of rates of growth. We will discuss them later in Section 1.1.

Since the subspaces corresponding to a rate of growth and combinations of them are invariant, the question of existence of invariant objects for the full system related to these linear spaces naturally arises. In particular, we may be interested in the spaces that converge the slowest, since these slowest convergences will dominate the long-term behavior.

The goal of this paper is to show that, under appropriate non-resonance conditions for the rates of growth, indeed one can find smooth manifolds tangent to the spaces invariant under the linearization. We also give examples that show that the non-resonance conditions are necessary for the existence of such invariant smooth manifolds.

Received by the editors April 9, 2003 and, in revised form, May 11, 2004.

2000 Mathematics Subject Classification. Primary 37D10, 37D25, 34D09, 70K45.

Key words and phrases. Lyapunov exponents, invariant manifolds, resonaces, normal forms. 
For the case of a fixed point of a local diffeomorphism of a Banach space near a fixed point, invariant manifolds associated to non-resonant subsets of the spectrum have been considered in dlL97. (see also ElB01, CFdlL03a, CFdlL03b, dlL03). Particular cases of non-resonant manifolds for uniformly hyperbolic systems were considered in Pes73, dlLW95, JPdIL95.

As remarked in Section 8.3 of dIL97 and in Section 2 of CFdlL03a, the results for fixed points imply results for more general sets using the device of lifting (see HP70]). Given a dynamical system $f$ we consider the action $\tilde{f}$ on the Banach space of $C^{0}$ vector fields defined by

$$
[\tilde{f} v](x)=\exp _{x}^{-1} f\left(\exp _{f^{-1}(x)} v\left(f^{-1}(x)\right)\right) .
$$

The zero vector field is, clearly, a fixed point of $\tilde{f}$. Moreover, the linearization of $\tilde{f}$ at the zero field is $f_{*}$, the push forward of $f$ acting on $C^{0}$ vector fields. Hence, under assumptions on the spectrum of $f_{*}$, we can associate invariant manifolds to $\tilde{f}$. It was shown in Mat68 that the spectral properties of $f_{*}$ are related to the growth rates of the linearized system.

In this paper, we develop a theory of non-resonant manifolds for orbits that satisfy some rather weak notion of hyperbolicity. This notion is based on properties of each individual orbit and does not require the uniformity assumptions that are required in the lifting approach. Indeed, we introduce a very weak notion of rates of growth (see Definition 1.2 below) which generalizes at the same time the notion of exponential dichotomies and the notion of Lyapunov exponents of non-uniform hyperbolic theory. Since the notion we consider also encompasses the notion of rates of growth in Oseledec's theorem Ose68, Rue79, the results also apply to random dynamical systems (see Section 1.1).

We note that the non-resonant conditions we will consider in this paper are automatically satisfied by the most contractive part of the stable spectrum. Hence, the results here generalize the classical results on strong stable manifolds. Nevertheless, in contrast with the strong stable manifolds, the non-resonant manifolds constructed here do not integrate to foliations (see [JPdlL95]).

For the sake of simplicity, we will formulate the results only for dynamical systems with discrete time. Analogous results are true for flows. The results for continuous time follow by taking time one maps of the flows. Of course, it is possible to give a direct proof of the results for flows following the arguments presented here for diffeomorphisms.

As a motivation for the study of the manifolds considered here, we will mention that they give one possible precise meaning to the idea of slow manifold which is used in many heuristic calculations of asymptotic behavior in dynamics; see Fra88, MP92 for a discussion of several possible meanings of slow manifold. From a mathematical point of view, we point out that one can prove, following the arguments in dILMM86, that the solutions of cohomology equations are smooth on the manifolds considered here. The fact that the manifolds considered here do not lead to a foliation provides an obstruction to smooth equivalence of dynamical systems which is not related to periodic orbits and which is not captured by non-autonomous linearization (see dlL92, JPdlL95).

1.1. Notions of rates of growth. There are two widely used methods to formalize the rates of growth of vectors along an orbit. 
One possibility — considered in [SS74, Fen74, Fen77] — is to require that there is a uniform expansion or contraction, but that there is a spread on the rates.

That is, for $\mu_{1} \leq \lambda_{1}<\mu_{2} \leq \lambda_{2}<\cdots<\mu_{p} \leq \lambda_{p}$, one characterizes the bundles $E_{x}^{i}$ by

$$
v \in E_{x}^{i} \Longleftrightarrow\left|D f^{n}(x) v\right| \leq \begin{cases}C \exp \left(\lambda_{i} n\right)|v|, & n \geq 0 \\ C \exp \left(\mu_{i} n\right)|v|, & n \leq 0\end{cases}
$$

We note that a consequence of (2) is that the angle between the different spaces $E_{x}^{i}$ is bounded from below.

Remark 1.1. It was shown in Mat68] that if the spectrum of $f_{*}$ - the push-forward by $f$ - acting on $C^{0}$ vector fields is contained in annuli of outer radius $e^{\lambda_{i}}$ and inner radius $e^{\mu_{i}}$, then, every orbit satisfies (2).

Under condition (2), it is possible to develop a theory of invariant manifolds and foliations based on lifting to actions of bundles as in (11). See [HP70, HPS77] for the origins of the theory and CFdlL03a, CFdlL03b for results for non-resonant invariant manifolds. In this paper, we will base our study on properties of individual orbits rather than on assuming spectral properties of operators acting on bundles.

Another characterization of rates of growth is based on the existence of Lyapunov exponents considered in Ose68, Rue79]:

$$
v \in E_{x}^{i} \Longleftrightarrow \lim _{n \rightarrow \pm \infty} \frac{1}{n} \ln \left(\left|D f^{n}(x) v\right|\right)=\lambda_{i}
$$

for some real numbers $\lambda_{1}, \ldots, \lambda_{p}$.

Note that definition (3) ignores polynomial terms in the rate and it can be quite non-uniform along the orbit. As is well known, Oseledec's theorem ensures that, given a measure $\rho$ invariant under the system, one has (3) $\rho$-almost everywhere and, moreover, one can find sets of measure arbitrarily close to full measure where there is some uniformity in the deterioration of the hyperbolicity properties.

One can consider that condition (2) requires that there is an exponential rate which is uniform along the orbit, but one has to allow a spread on the rate. On the other hand, in condition (3), there is an exponential rate, but one only requires that the exponential rate, happens in an averaged sense and that it does not need to be uniform along the orbit.

Neither of the characterizations of rates of growth (2) and (3) is more general than the other. Even if a vector is in one of the subbundles in (2), it may fail to have a Lyapunov exponent if the rates of growth keep on oscillating. Such points are easy to construct in hyperbolic systems (e.g. horseshoes) that have a symbolic dynamics. On the other hand, systems admitting Lyapunov exponents may have fluctuations that destroy the possibility of uniformity.

We introduce a new definition that encompasses both of the previous definitions (2) and (3). We allow the existence of a spread in the exponential rate as well as a deterioration of the constants along the orbit.

Definition 1.2. Given $\lambda=\left\{\lambda_{1}, \ldots, \lambda_{p}\right\}$ and $\mu=\left\{\mu_{1}, \ldots, \mu_{p}\right\}$ such that $\mu_{1} \leq \lambda_{1}<$ $\mu_{2} \leq \lambda_{2}<\cdots<\mu_{p} \leq \lambda_{p}, \ell>1, \varepsilon>0$, we say that a point $x$ has a $(\lambda, \mu, \varepsilon, \ell)$ regular orbit if we can find invariant decompositions $T_{f^{m}(x)} M=\bigoplus_{i=1}^{p} E_{f^{m}(x)}^{i}$ such 
that:

(i) $v \in E_{f^{m}(x)}^{i}$ implies

$$
\ell^{-1} \exp \left(\mu_{i} n-\varepsilon|m|\right)|v| \leq\left|D f^{n}\left(f^{m}(x)\right) v\right| \leq \ell \exp \left(\lambda_{i} n+\varepsilon|m|\right)|v|
$$

for $n \geq 0$, and

$$
\ell^{-1} \exp \left(\lambda_{i} n-\varepsilon|m|\right)|v| \leq\left|D f^{n}\left(f^{m}(x)\right) v\right| \leq \ell \exp \left(\mu_{i} n+\varepsilon|m|\right)|v|
$$

for $n<0$.

(ii) angle $\left(E_{f^{m}(x)}^{i}, E_{f^{m}(x)}^{j}\right) \geq \ell^{-1} \exp (-\varepsilon|m|), \quad i \neq j$.

Denote $I_{i}=\left[\mu_{i}, \lambda_{i}\right]$. Also denote by $\Lambda_{\lambda, \mu, \varepsilon, \ell}$ the set of $(\lambda, \mu, \varepsilon, \ell)$-regular orbits.

We recall that if $f$ is a $C^{1}$ system and $\rho$ is an ergodic invariant probability measure, Oseledec's multiplicative ergodic theorem implies that, if the Lyapunov exponents are $\gamma_{i}, i=1, \ldots, p$, then, for $\varepsilon>0$, the sets $\Lambda_{\gamma-\varepsilon, \gamma+\varepsilon, \varepsilon, \ell}$ can be made to have measure as close to full as desired by choosing $\ell$ big enough. That is, $\rho$-almost all orbits are regular, but the constant $\ell$ cannot be chosen uniformly. The sets $\Lambda_{\gamma+\varepsilon, \gamma-\varepsilon, \varepsilon, \ell}$ are often called Pesin sets.

Condition (2) clearly implies Definition 1.2 taking $\varepsilon=0$ and $\ell$ a suitable large number. A fortiori, it is shown in Mat68] that the fact that the spectrum of the push-forward is contained in annuli of inner radii $\exp \mu_{i}$ and outer radii $\exp \lambda_{i}$ is equivalent to the fact that all orbits satisfy Definition 1.2 with $\varepsilon=0$ and $\ell$ chosen uniformly for all points.

1.2. Non-resonance. Given two intervals $I_{1}, I_{2} \subset \mathbb{R}$, we denote

$$
I_{1}+I_{2}=\left\{t=t_{1}+t_{2}: t_{1} \in I_{1}, t_{2} \in I_{2}\right\} .
$$

Of course, when the intervals consist of one number, the above operation corresponds to the sum of numbers.

Definition 1.3. Let $\left\{I_{i}\right\}_{i=1}^{p}$ be a collection of intervals. Given $S \subset\{1,2, \ldots, p\}$, we say that a subset $\left\{I_{i}\right\}_{i \in S}$ is non-resonant if for $j \in \mathbb{N}, j \geq 2$, and any collection $i_{1}, \ldots, i_{j} \in S$ of indices (perhaps repeated), we have

$$
\left(I_{i_{1}}+\cdots+I_{i_{j}}\right) \cap \bigcup_{i \in S^{c}} I_{i}=\emptyset .
$$

We say that a subset $\left\{I_{i}\right\}_{i \in S}$ is contractive when

$$
\bigcup_{i \in S} I_{i} \subset \mathbb{R}^{-} .
$$

Denote $I^{S}=\bigcup_{i \in S} I_{i}$ and $I^{S^{c}}=\bigcup_{i \in S^{c}} I_{i}$.

Denote $E_{x}^{S}=\bigoplus_{i \in S} E_{x}^{i}$ and $E_{x}^{S^{c}}=\bigoplus_{i \in S^{c}} E_{x}^{i}$.

We clearly have $T_{x} M=E_{x}^{S} \oplus E_{x}^{S^{c}}$.

Denote $\Pi_{x}^{S}$ and $\Pi_{x}^{S^{c}}$ as the projections associated to this decomposition. We use the abbreviations $d_{S}(x)=\operatorname{dim} E_{x}^{S}$ and $d_{S^{c}}(x)=\operatorname{dim} E_{x}^{S^{c}}$.

For collections of intervals $\left\{I_{i}\right\}$ and subsets $S$ satisfying (6) and (77), we denote by $N_{S}$ the integer defined by

$$
N_{S}=\left[\frac{\min \left\{t \in I^{S^{c}}\right\}}{\max \left\{t \in I^{S}\right\}}\right],
$$

where $[t]$ denotes integer part of $t$. 
It is clear that for collections satisfying (7), condition (6) is automatically verified if $j>\max \left\{N_{S}, 0\right\}$.

Remark 1.4. Note that in part (i) of Definition 1.2 we have only assumed that in the invariant space $E_{x}^{i}$ we have the rates of growth (4), (5). In the literature, it is often assumed that the space $E_{x}^{i}$ is precisely characterized by (4), (5).

One interesting example of spaces where assumption (i) of Definition 1.2 applies but which is not characterized by (4), (5) is Cartesian product systems in which the two factors overlap. For example, if we take the Cartesian product of a system by itself, $F(x, y)=(f(x), f(y))$ and $E_{x}^{i}$ is a spectral decomposition for $f$, we see that $\tilde{E}_{(x, y)}^{i}=E_{x}^{i} \times\{0\}$ is a space admissible for our definition. However, the only spectral space is $\left(E_{x}^{i} \times\{0\}\right) \oplus\left(\{0\} \times E_{y}^{i}\right)$. It is perfectly possible to have examples such as those mentioned here that satisfy the non-resonance conditions in Definition 1.3. Note that there we are only assuming that (6) happens for $j \geq 2$.

Remark 1.5. For collections of intervals which satisfy (7), property (6) amounts to a finite number of conditions. It is clear that if we slightly enlarge the intervals both conditions will remain valid.

Remark 1.6. When the intervals are points, it is instructive to compare condition (6) with the conditions of Sternberg's linearization theorem. The conditions of Sternberg's linearization theorem require that no interval contains sums of points in other intervals. Here we only require that the numbers of the set $I^{S^{c}}$ cannot be obtained as sums of numbers in the set $I^{S}$.

Indeed, the proof presented here has some similarities with the proof of Sternberg's theorem. We start by computing a polynomial approximation to the desired object and then use a contraction argument to show that the very approximate polynomial solution can be modified to become a true solution of the problem.

A Sternberg's type theorem along orbits under full non-resonance conditions can be found in Yom88. Similar results are crucial for [Yom87. A related theory is the one of the non-autonomous normal forms GK98 developed under uniformity conditions on the bundle. It seems clear that one could work out a similar theory under assumptions on the behavior of individual orbits.

Remark 1.7. An important case of a non-resonant set is when $S$ includes the $I_{i}$ contained in $(-\infty, l)$, where $l<0$ and $l \notin \bigcup_{i=1}^{p} I_{i}$. In such a case, the bundle $E_{x}^{S}$ is the strongly stable bundle, and our results give the usual strongly stable invariant manifold.

Note that the strong stable manifold admits the characterization

$$
W_{x}^{s s}=\left\{y: d\left(f^{n}(x), f^{n}(y)\right) \leq C_{y, x} \lambda^{n}\right\}
$$

which makes it clear that $y \in W_{x}^{s s}$ is an equivalence relation and that, therefore, the set of strong stable manifolds is a lamination.

More general non-resonant manifolds do not admit a characterization in terms of rates of growth. In [JPdlL95] one can find examples where the non-resonant manifolds of neighboring points have non-trivial intersections.

\subsection{Statement of main results.}

Definition 1.8. We will say that a family of maps $\left\{w_{n}\right\}_{n \in \mathbb{Z}}$ is uniformly $C^{k}$ if the maps are $C^{k}$ and $\sup _{n \in \mathbb{Z}}\left\|w_{n}\right\|_{C^{k}}<\infty$. We will also say that a family of parameterized manifolds $\left\{W_{n}\right\}_{n \in \mathbb{Z}}$ is uniformly $C^{k}$ if there are parameterizations of $W_{n}$ that form a uniformly $C^{k}$ family. 
The main result of this paper is:

Theorem 1.9. Let $f$ be a $C^{r}$ diffeomorphism, $r \in \mathbb{N} \cup\{\infty\}, r \geq 2$, of a compact $C^{\infty}$ finite-dimensional manifold $M$. Let $x \in \Lambda_{\lambda, \mu, \varepsilon, \ell}, I_{i}=\left[\mu_{i}, \lambda_{i}\right], i \in\{1, \ldots, p\}$, and $\left\{I_{i}\right\}_{i \in S}, S \subset\{1, \ldots, p\}$, be a non-resonant and contractive set. Assume that $r \geq N_{S}+1$ and that $\varepsilon<\delta / 2$ and $\delta$ is small enough such that if we consider the enlarged intervals $\tilde{I}_{i}=I_{i}+[-2 \delta, 2 \delta]$, the set $\left\{\tilde{I}_{i}\right\}_{i \in S}$ is still a non-resonant and contractive set.

Then, there exist maps $w_{n}: B(0,1) \subset \mathbb{R}^{d_{S}} \longrightarrow M$, where $B(0,1)$ is the unit ball in $\mathbb{R}^{d_{S}}, n \in \mathbb{Z}$, in such a way that

(a) $w_{n}(0)=f^{n}(x)$.

(b) $w_{n}$ are uniformly $C^{r}$.

If we denote by $W_{n}$ the range of $w_{n}$, then:

(c) $f\left(W_{n}\right) \subset W_{n+1}$.

(d) $T_{f^{n}(x)} W_{n}=E_{f^{n}(x)}^{S}$.

Also, there exists $K>0$ such that the manifolds $W_{n}$ contain a disk of radius

$$
K \ell^{-2} \sqrt{\tanh \delta} \exp (-(2 \varepsilon+\delta)|n|) .
$$

Moreover, if $W_{n}, \hat{W}_{n}$ are families of manifolds satisfying (c) and (d), then:

(e) If $W_{n}, \hat{W}_{n}$ are uniformly $C^{m}$ for some $m \leq r$, then $T_{f^{n}(x)}^{i} W_{n}=T_{f^{n}(x)}^{i} \hat{W}_{n}$ for $i \leq m$.

(f) If $W_{n}, \hat{W}_{n}$ are uniformly $C^{k}$ for some $k>N_{S}$, then $W_{n} \cap B_{n}=\hat{W}_{n} \cap B_{n}$ for some balls $B_{n}$ around $f^{n}(x)$.

In particular, if the manifolds are uniformly $C^{N_{S}+1}$, they have to agree with the manifolds range $w_{n}$ and hence they are uniformly $C^{r}$.

(g) If $\sup _{n \in \mathbb{Z}}\left\|W_{n}\right\|_{C^{N_{s}}}<\infty$ and $W_{0}$ is a $C^{k}$ manifold for some $N_{S}<k \leq r$, then $\sup _{n \in \mathbb{N}}\left\|W_{-n}\right\|_{C^{k}}<\infty$.

The meaning of the above result is that if the set $S$ of rates of growth is nonresonant, we can find a collection of smooth manifolds that are non-linear analogues of the linear subspaces invariant under the linearized map.

The final conclusions of Theorem 1.9 are uniqueness conclusions that say that these systems of leaves are unique under some regularity properties.

Part (e) says that the $m$-jets of the manifolds are uniquely determined provided they are uniformly $C^{m}$.

Roughly speaking, part (f) tells us that the manifolds are unique when they are regular beyond a critical value. In particular, when we know that they are more regular than this critical value, they are as regular as the map. Hence, part (f) is a bootstrap of the regularity argument which starts working when the regularity is higher than a critical value related to rates of contraction. Similar bootstrap arguments appear in rigidity theory (see, for example, dlL92]).

In Section 3, we provide an example that illustrates the role of the critical regularity in the uniqueness properties.

It is important to remark that the proof will only use constructions in a neighborhood of an orbit.

Remark 1.10. We have formulated Theorem 1.9 only for maps on finite-dimensional manifolds. Nevertheless, there are versions along the same lines for maps on infinitedimensional Banach spaces which have smooth cut-off functions (e.g. Hilbert 
spaces). However, we note that some of the arguments we present - notably the construction of the Lyapunov metric - require some serious modifications (it is somewhat easier for Hilbert spaces). We will not consider these cases in this paper.

Remark 1.11. Even in the case that the orbit is a fixed point, the non-resonance condition is necessary for the existence of an invariant manifold. As we will see, the candidates for a jet satisfy functional equations which may fail to have solutions if the non-resonance conditions are violated. Hence, in those examples where there is no candidate for a jet, there cannot be a smooth invariant manifold. We refer to Example 5.5 of dlL97 for more details.

Remark 1.12. Note that, in contrast with many of the results in uniform normal hyperbolicity which are valid for $r \geq 1$, our results are only valid for $r \geq 2$. It seems to us that the proof will work for $r=1+\delta$ with mainly notational difficulties. Nevertheless, the proof with $r=1$ does not work in general since the paper Pug84 contains an example of a $C^{1}$ system satisfying Definition 1.2 for which there are no $C^{1}$ invariant manifolds tangent to the spaces. The paper above contains the conjecture that in the case that the stable manifold is one dimensional, one could get stable manifolds even for $r=1$.

Remark 1.13. If one has hyperbolicity properties for all the orbits, then one could hope that the results given here for individual orbits could be made coherent to integrate the distribution $E_{x}^{S}$ to give a foliation. For the stable foliation, such a procedure is carried out in [Fen74, Fen77]. Nevertheless, in the generality considered here, the leaves produced in this paper do not integrate to a foliation. Even in the very uniform case when $\varepsilon=0$, one can find examples $-C^{\omega}$ close to linear automorphisms of the torus - where these invariant manifolds cross in arbitrarily small neighborhoods (see [JPdlL95]).

In some particular cases - e.g. maps of the torus close to linear and when $S$ corresponds to intervals contained in $\mathbb{R}^{-}$- there is a way of integrating the foliations based not on local properties but on global behavior (see dlLW95). The leaves produced in dlLW95 are not very smooth and, hence, are very different from the ones considered here. In particular, in the proof given in dlLW95 one has to take into account global properties of the manifold to obtain that the leaves integrate to a foliation.

Remark 1.14. The results of Theorem 1.9 are local. The proof consists of examining the sequence of maps $f_{n}$ that are coordinate representations of $f$ from a neighborhood of $x_{n}$ to a neighborhood of $x_{n+1}$. Indeed, we deduce Theorem 1.9 from Theorem 2.5. which asserts the existence of invariant manifolds for sequences of maps in such a way that the linearization is non-resonant.

Besides the situation considered in Theorem [1.9, there are other cases where Theorem 2.5 appears naturally. Notably, if $f_{n}$ is a random sequence of maps, Oseledec's theorem Ose68, Rue79] shows that almost all orbits admit Lyapunov exponents. Hence, for random dynamical systems, provided that the Lyapunov exponents satisfy the non-resonance assumptions, we obtain that the manifolds produced in Theorem 1.9 exist with probability 1.

Studies of non-autonomous Sternberg theorems for random systems have been undertaken in the preprint [LL04, which appeared after this paper was submitted. 


\section{Proof of Theorem 1.9}

The proof of Theorem 1.9 starts very similar to the proof of the invariant manifold theorem in Pes76, Pes77] and subsequent papers. We start by defining a Lyapunov metric, which is singular with respect to the Euclidean metric but makes the hyperbolicity properties of the problem uniform. This allows us to choose a convenient system of coordinates in neighborhoods of each of the points in the orbit.

In a second step, we see that by writing the manifolds in the form $W_{n}=$ graph $V_{n}$ for certain functions $V_{n}$, under the non-resonance conditions, it is possible to uniquely determine candidates $V_{n}^{0}$ for the jet of the functions $V_{n}$. That is, if the functions $V_{n}$ were differentiable enough, we could take derivatives of the invariance equation and obtain functional equations satisfied by the sequence of jets. We will show that these functional equations admit unique solutions. We emphasize that at this point we only require the non-resonance condition (6) and not the contractive hypothesis (7).

In a third and final step, we use the computed candidates for jets to show that we can transform the equation satisfied by $U_{n}:=V_{n}-V_{n}^{0}$ into an equation that can be treated with the contraction mapping principle in some appropriate spaces by using assumption (7).

The motivation for this scheme is that for functions that vanish at order $N_{S}$ at the origin, a contraction $\lambda$ on the right contracts the norms based on derivatives of order $N_{S}$ by $\lambda^{N_{S}}$. If $N_{S}$ is large enough, this contraction factor can overcome the expansion factors generated by the other directions.

Note that one of the consequences of this study will be that the $U_{n}$ vanish to sufficiently high order at the origin so that the $V_{n}^{0}$ are indeed the jets of the $V_{n}$.

2.1. The Lyapunov metric and coordinates around the orbit. The main goal of this section is to establish Lemma 2.1] which provides us with a system of coordinates around an orbit. The main idea - rather standard in the study of nonuniformly hyperbolic systems - is that one can define a Lyapunov metric around an orbit which makes the hyperbolicity uniform. Once we express all the properties in this metric, many of the methods of the theory of uniformly hyperbolic systems start to apply. In our case, we will reduce the problem to the study of systems with exponential dichotomies.

We will use different norms and scalar products. The subindices E, L, R will stand for Euclidean, Lyapunov and Riemannian norms or scalar products, respectively. We also recall that, given a set $D$, the modulus of continuity of $h_{\mid D}$ is

$$
\omega(h, \eta)=\sup _{y, z \in D,|y-z| \leq \eta}\|h(y)-h(z)\| .
$$

Lemma 2.1. Let $M$ be a compact $C^{\infty}$ d-dimensional manifold. Given a $C^{r}$ map $f: M \rightarrow M, r \in \mathbb{N} \cup\{\infty\}, r \geq 2, x \in \Lambda_{\lambda, \mu, \varepsilon, \ell}, \delta>2 \varepsilon, \tau>0$ and a fixed (Euclidean) orthogonal decomposition $\mathbb{R}^{d}=\bigoplus_{i=1}^{p} E^{i}$ such that $\operatorname{dim} E^{i}=\operatorname{dim} E_{f^{k}(x)}^{i}$, there exists a sequence of $C^{\infty}$ maps

$$
\Phi_{k}: B(0,1) \subset \mathbb{R}^{d} \rightarrow M, \quad k \in \mathbb{Z},
$$

such that

(i) $\Phi_{k}(0)=f^{k}(x)$.

(ii) $D \Phi_{k}(0) E^{i}=E_{f^{k}(x)}^{i}$. 
If we denote by $f_{k}=\Phi_{k+1}^{-1} \circ f \circ \Phi_{k}$ we have:

(iii) $\exp \left(\mu_{i}-2 \delta\right)\|v\|_{E} \leq\left\|D f_{k}(0) v\right\|_{E} \leq \exp \left(\lambda_{i}+2 \delta\right)\|v\|_{E}$ for $v \in E^{i}$.

(iv) range $\left(\Phi_{k}\right) \supset\left\{y: d_{R}\left(f^{k}(x), y\right) \leq(2 / \pi) \Gamma p^{-1 / 2} \ell^{-2} \sqrt{\tanh \delta} \exp (-(2 \varepsilon+\delta)|k|)\right\}$, where $\Gamma$ is some positive constant.

Moreover, if $r<\infty$,

(v) $\sup _{k \in \mathbb{Z},|x|<1,2 \leq j \leq r}\left\|D^{j} f_{k}(x)\right\| \leq \tau$.

(vi) The modulus of continuity of $D^{r} f_{k}$ is bounded independently of $k$ by $\tau$.

Proof. For a regular orbit we define the Lyapunov inner product in $E_{f^{k}(x)}^{i}$ by

$$
\begin{aligned}
\langle v, w\rangle_{L}= & \sum_{n \geq 0} e^{-2\left(\lambda_{i}+\delta\right) n}\left\langle D f^{n}\left(f^{k}(x)\right) v, D f^{n}\left(f^{k}(x)\right) w\right\rangle_{R} \\
& +\sum_{n<0} e^{-2\left(\mu_{i}-\delta\right) n}\left\langle D f^{n}\left(f^{k}(x)\right) v, D f^{n}\left(f^{k}(x)\right) w\right\rangle_{R}
\end{aligned}
$$

for $v, w \in E_{f^{k}(x)}^{i}$, and in $T_{f^{k}(x)} M$

$$
\langle v, w\rangle_{L}=\sum_{i=1}^{p}\left\langle\Pi_{f^{k}(x)}^{i} v, \Pi_{f^{k}(x)}^{i} w\right\rangle_{L}, \quad v, w \in T_{f^{k}(x)} M,
$$

where $\Pi_{f^{k}(x)}^{i}$ are the projections onto $E_{f^{k}(x)}^{i}$. Note that the subspaces $E_{f^{k}(x)}^{i}$ are orthogonal with respect to the Lyapunov metric, and that, by Definition 1.2 , denoting by $\varphi_{k}$ the minimum angle between the subspaces $E_{f^{k}(x)}^{i},\left\|\Pi_{f^{k}(x)}^{i}\right\| \leq$ $\frac{1}{\sin \varphi_{k}} \leq \frac{\pi}{2 \varphi_{k}} \leq \frac{\pi}{2} \ell \exp (\varepsilon|k|)$. By the previous definitions, if $v \in E_{f^{k}(x)}^{i}$,

$$
\begin{aligned}
\|v\|_{L}^{2} & \leq \sum_{n \geq 0} e^{-2\left(\lambda_{i}+\delta\right) n}\left\|D f^{n}\left(f^{k}(x)\right) v\right\|_{R}^{2}+\sum_{n<0} e^{-2\left(\mu_{i}-\delta\right) n}\left\|D f^{n}\left(f^{k}(x)\right) v\right\|_{R}^{2} \\
& \leq\left[\sum_{n \geq 0} e^{-2\left(\lambda_{i}+\delta\right) n} \ell^{2} e^{2 \lambda_{i} n} e^{2 \varepsilon|k|}+\sum_{n<0} e^{-2\left(\mu_{i}-\delta\right) n} \ell^{2} e^{2 \mu_{i} n} e^{2 \varepsilon|k|}\right]\|v\|_{R}^{2} \\
& =\left(\left(\ell e^{\varepsilon|k|}\right)^{2} / \tanh \delta\right)\|v\|_{R}^{2}
\end{aligned}
$$

and if $v \in T_{f^{k}(x)} M$,

$$
\|v\|_{L}^{2}=\sum_{i=1}^{p}\left\|\Pi_{f^{k}(x)}^{i} v\right\|_{L}^{2} \leq\left(\sum_{i=1}^{p} \frac{\pi^{2}}{4} \ell^{4} e^{4 \varepsilon|k|} / \tanh \delta\right)\|v\|_{R}^{2}
$$

and hence

$$
\|v\|_{L} \leq\left(\sqrt{p}(\pi / 2) \ell^{2} e^{2 \varepsilon|k|} / \sqrt{\tanh \delta}\right)\|v\|_{R} .
$$

Next, we define a family of linear maps $C_{k}$.

Given the orthogonal decomposition $\mathbb{R}^{d}=\bigoplus_{i=1}^{p} E^{i}$ we define $C_{k \mid E^{i}}: E^{i} \rightarrow$ $E_{f^{k}(x)}^{i}$ as follows. We take an orthonormal basis $\left\{e_{1}^{i}, \ldots, e_{d_{i}}^{i}\right\}$ in each $E^{i}$ with respect to the Euclidean scalar product and an orthonormal basis $\left\{u_{1}^{i}, \ldots, u_{d_{i}}^{i}\right\}$ in each $E_{f^{k}(x)}^{i}$ with respect to $\langle\cdot, \cdot\rangle_{L}$. We just define $C_{k \mid E^{i}}$ by the relations $C_{k} e_{j}^{i}=$ $u_{j}^{i}, 1 \leq j \leq d_{i}, 1 \leq i \leq p$. With this definition $C_{k}$ becomes an isometry from $\left(\mathbb{R}^{d},\|\cdot\|_{E}\right)$ to $\left(T_{f^{k}(x)} M,\|\cdot\|_{L}\right)$. Indeed, $\left\|C_{k}\left(\sum \lambda_{j} e_{j}\right)\right\|_{L}^{2}=\left\|\sum \lambda_{j} u_{j}\right\|_{L}^{2}=\sum\left|\lambda_{j}\right|^{2}=$ 
$\left\|\sum \lambda_{j} e_{j}\right\|_{E}^{2}$. Moreover, from (11) we have

$$
\left\|C_{k}^{-1} v\right\|_{E}=\|v\|_{L} \leq \frac{\sqrt{p}(\pi / 2) \ell^{2} e^{2 \varepsilon|k|}}{\sqrt{\tanh \delta}}\|v\|_{R}
$$

We also have

$$
\begin{aligned}
\left\|C_{k} v\right\|_{R} & \leq \sum_{i=1}^{p}\left\|\Pi_{f^{k}(x)}^{i} C_{k} v\right\|_{R} \leq \sum_{i=1}^{p}\left\|\Pi_{f^{k}(x)}^{i} C_{k} v\right\|_{L} \\
& \leq \sqrt{p}\left(\sum_{i=1}^{p}\left\|\Pi_{f^{k}(x)}^{i} C_{k} v\right\|_{L}^{2}\right)^{1 / 2}=\sqrt{p}\left\|C_{k} v\right\|_{L}=\sqrt{p}\|v\|_{E} .
\end{aligned}
$$

Let exp be the exponential map of the Riemannian metric in $M$. We define

$$
\Phi_{k}(y)=\exp _{f^{k}(x)}\left(\gamma_{k} C_{k} y\right),
$$

and we claim that if we choose $\gamma_{k}$ in a suitable way, $\Phi_{k}$ satisfies all the conclusions of Lemma 2.1.

Indeed, conclusions (i) and (ii) are immediately satisfied. Since $D \Phi_{k}(0)=\gamma_{k} C_{k}$, $D \Phi_{k}(0)$ transforms the Euclidean metric into $\gamma_{k}$ times the Lyapunov metric.

We have

$$
\begin{aligned}
D f_{k}(0) & =D \Phi_{k+1}^{-1}\left(f^{k+1}(x)\right) D f\left(f^{k}(x)\right) D \Phi_{k}(0) \\
& =\frac{1}{\gamma_{k+1}} C_{k+1}^{-1} D f\left(f^{k}(x)\right) \gamma_{k} C_{k}
\end{aligned}
$$

and if $v \in E^{i}$

$$
\left\|D f_{k}(0) v\right\|_{E}=\frac{\gamma_{k}}{\gamma_{k+1}}\left\|D f\left(f^{k}(x)\right) C_{k} v\right\|_{L}
$$

Furthermore, if $v \in E^{i}$

$$
\begin{aligned}
\| D f( & \left.f^{k}(x)\right) C_{k} v \|_{L}^{2} \\
= & \sum_{n \geq 0} e^{-2\left(\lambda_{i}+\delta\right) n}\left\|D f^{n}\left(f^{k+1}(x)\right) D f\left(f^{k}(x)\right) C_{k} v\right\|_{R}^{2} \\
& \quad+\sum_{n<0} e^{-2\left(\mu_{i}-\delta\right) n}\left\|D f^{n}\left(f^{k+1}(x)\right) D f\left(f^{k}(x)\right) C_{k} v\right\|_{R}^{2} \\
= & \sum_{n \geq 0} e^{-2\left(\lambda_{i}+\delta\right) n}\left\|D f^{n+1}\left(f^{k}(x)\right) C_{k} v\right\|_{R}^{2} \\
& +\sum_{n<0} e^{-2\left(\mu_{i}-\delta\right) n}\left\|D f^{n+1}\left(f^{k}(x)\right) C_{k} v\right\|_{R}^{2} \\
= & e^{2\left(\lambda_{i}+\delta\right)}\left[\sum_{n \geq 0} e^{-2\left(\lambda_{i}+\delta\right) n}\left\|D f^{n}\left(f^{k}(x)\right) C_{k} v\right\|_{R}^{2}-\left\|C_{k} v\right\|_{R}^{2}\right] \\
& \quad+e^{2\left(\mu_{i}-\delta\right)}\left[\sum_{n<0} e^{-2\left(\mu_{i}-\delta\right) n}\left\|D f^{n}\left(f^{k}(x)\right) C_{k} v\right\|_{R}^{2}+\left\|C_{k} v\right\|_{R}^{2}\right] \\
\leq & e^{2\left(\lambda_{i}+\delta\right)}\left\|C_{k} v\right\|_{L}^{2} .
\end{aligned}
$$

Then

$$
\left\|D f_{k}(0) v\right\|_{E} \leq \frac{\gamma_{k}}{\gamma_{k+1}} e^{\left(\lambda_{i}+\delta\right)}\left\|C_{k} v\right\|_{L} .
$$

Recall that $\left\|C_{k} v\right\|_{L}=\|v\|_{E}$. This proves the upper bound claimed in (iii). 
The proof of the lower bound is completely analogous. Following the previous computations we have

$$
\left\|D f\left(f^{k}(x)\right) C_{k} v\right\|_{L} \geq e^{\mu_{i}-\delta}\left\|C_{k} v\right\|_{L}
$$

and hence

$$
\exp \left(\mu_{i}-\delta\right) \frac{\gamma_{k}}{\gamma_{k+1}}\|v\|_{E} \leq\left\|D f_{k}(0) v\right\|_{E} \leq \exp \left(\lambda_{i}+\delta\right) \frac{\gamma_{k}}{\gamma_{k+1}}\|v\|_{E}
$$

We take $\gamma_{k}=\Gamma e^{-\delta|k|}$ with $0<\Gamma \leq 1$ to be determined later on. Then, if $k \geq 0$, $\frac{\gamma_{k}}{\gamma_{k+1}}=e^{\delta}$ and, if $k<0, \frac{\gamma_{k}}{\gamma_{k+1}}=e^{-\delta}$.

To prove (iv), we observe that $\gamma_{k} C_{k}$ sends the unit ball of $\mathbb{R}^{d}$ to $\gamma_{k}$ times the unit ball of $T_{f^{k}(x)} M$ with respect to the Lyapunov metric. From (11) the unit Lyapunov ball contains the Riemannian ball of radius $\gamma_{k} \frac{2}{\sqrt{p} \pi} \ell^{-2} e^{-2 \varepsilon|k|} \sqrt{\tanh \delta}$ and finally $\exp _{f^{k}(x)}$ sends this ball to $M$.

For (v) we evaluate the derivative $D^{r} f_{k}=D^{r}\left(\Phi_{k+1}^{-1} \circ f \circ \Phi_{k}\right)$. Recall the Faa di Bruno formulas

$$
D^{r} f_{k}=\sum_{j=1}^{r} \sum_{\substack{l_{1}+\cdots+l_{j}=r \\ 1 \leq l_{1}, \ldots, l_{j} \leq r}} c_{l_{1}, \ldots, l_{j}}^{r, j} D^{j} \Phi_{k+1}^{-1} \circ\left(f \circ \Phi_{k}\right) D^{l_{1}}\left(f \circ \Phi_{k}\right) \cdots D^{l_{j}}\left(f \circ \Phi_{k}\right)
$$

and

$$
D^{l}\left(f \circ \Phi_{k}\right)=\sum_{j=1}^{l} \sum_{\substack{m_{1}+\cdots+m_{j}=l \\ 1 \leq m_{1}, \ldots, m_{j} \leq l}} c_{m_{1}, \ldots, m_{j}}^{l, j} D^{j} f \circ \Phi_{k} D^{m_{1}} \Phi_{k} \cdots D^{m_{j}} \Phi_{k},
$$

where the $c$ 's are combinatorial coefficients which depend on the indices.

In our case, we were mainly interested in

$$
\Phi_{k}=\exp _{f^{k}(x)} \circ\left(\gamma_{k} C_{k}\right)
$$

and

$$
\Phi_{k+1}^{-1}=\frac{1}{\gamma_{k+1}} C_{k+1}^{-1} \exp _{f^{k+1}(x)}^{-1} .
$$

From now on $K$ will stand for a constant which only depends on the manifold $M$ and the properties of the exponential map.

We observe that

$$
\left\|D^{m} \Phi_{k}\right\| \leq K\left(\gamma_{k}\left\|C_{k}\right\|\right)^{m} \text {. }
$$

Similarly,

$$
\left\|D^{j} \Phi_{k+1}^{-1}\right\| \leq K \frac{1}{\gamma_{k+1}}\left\|C_{k+1}^{-1}\right\| .
$$

By (12) the norm of $C_{k}$ considered as a linear map from $\left(\mathbb{R}^{d},\|\cdot\|_{E}\right)$ to $\left(T_{f^{k+1}(x)} M\right.$, $\left.\|\cdot\|_{R}\right)$ is smaller than $\sqrt{p}$.

We deduce that

$$
\begin{aligned}
\left\|D^{r} f_{k}\right\| & \leq K \ell^{2} \frac{\gamma_{k}^{r}}{\gamma_{k+1}} \frac{e^{2 \varepsilon|k|}}{\sqrt{\tanh \delta}}\|f\|_{C^{r}} \\
& =\frac{K \ell^{2} \Gamma^{r-1}}{\sqrt{\tanh \delta}} e^{(2 \varepsilon-(r-1) \delta)|k|}\|f\|_{C^{r}} .
\end{aligned}
$$

Therefore if we take $\Gamma$ small enough we can assume without loss of generality that the derivatives of $f_{k}$ of order $r \geq 2$ are as small as desired. 
Finally we prove (vi). Since $M$ is compact, all derivatives of $f$ are uniformly continuous. Let $B_{z}$ be the ball of center 0 and radius $\sqrt{p} \Gamma$ in $T_{z} M$ (the radius $\sqrt{p} \Gamma$ is motivated by (12)). Since $M$ is $C^{\infty}$ and compact

$$
\sup _{0 \leq m \leq r+1} \sup _{z \in M} \sup _{x \in B_{z}}\left\|D^{m} \exp _{z}(x)\right\|<\infty .
$$

For $m \leq r$ and $\|y\|_{E},\|z\|_{E} \leq 1$ we have

$$
\left\|D^{m} \Phi_{k}(y)\right\|_{R}=\left\|D^{m} \exp _{f^{k}(x)}\left(\gamma_{k} C_{k} y\right)\left(\gamma_{k} C_{k}\right)^{\otimes m}\right\| \leq K \Gamma^{m} e^{-\delta|k| m}
$$

and

$$
\begin{aligned}
\left\|D^{m} \Phi_{k}(y)-D^{m} \Phi_{k}(z)\right\| & \leq\left\|D^{m+1} \exp _{f^{k+1}(x)}\right\|\left\|\gamma_{k} C_{k}\right\|^{m+1}\|y-z\| \\
& \leq K \Gamma^{m+1} e^{-\delta|k|(m+1)}\|y-z\| .
\end{aligned}
$$

Then by (14) and the fact that $D^{j} f, 0 \leq j \leq r$, are bounded and uniformly continuous, we get that

$$
\left\|D^{l}\left(f \circ \Phi_{k}\right)\right\| \leq K \Gamma^{l} e^{-\delta|k| l}\|f\|_{C^{l}}
$$

and

$$
\begin{aligned}
\omega\left(D^{l}\left(f \circ \Phi_{k}\right), \eta\right) & =\sum_{j=1}^{l} K \Gamma^{l} e^{-\delta|k| l} \omega\left(D^{j} f, K \Gamma \eta\right)+K \Gamma^{l+1} e^{-\delta|k|(l+1)}\|f\|_{C^{l}} \eta \\
& =K \Gamma^{l} e^{-\delta|k| l} o(1)+K \Gamma^{l+1} e^{-\delta|k|(l+1)} \eta .
\end{aligned}
$$

Next we consider the modulus of continuity of $D^{j} \Phi_{k+1}^{-1} \circ\left(f \circ \Phi_{k}\right)$. For simplicity, we just bound the modulus of continuity using the mean value theorem assuming that $D^{j+1} f$ is uniformly bounded. We get

$$
\omega\left(D^{j} \Phi_{k+1}^{-1} \circ\left(f \circ \Phi_{k}\right), \eta\right) \leq K \ell^{2} \frac{e^{\delta}}{\sqrt{\tanh \delta}} e^{2 \varepsilon|k+1|}\|f\|_{C^{1}} \eta .
$$

Finally by (13)

$$
\begin{aligned}
\omega\left(D^{r} f_{k}, \eta\right) & \\
\leq & K \ell^{2} \frac{e^{\delta}}{\sqrt{\tanh \delta}} e^{2 \varepsilon|k+1|} \Gamma^{r} e^{-\delta|k| r}\|f\|_{C^{1}}\|f\|_{C^{r}} \eta \\
& +K \sum_{j=1}^{r} \sum_{l_{j}} \Gamma^{-1} e^{\delta|k+1|} \ell^{2} \frac{e^{2 \varepsilon|k+1|}}{\sqrt{\tanh \delta}} \Gamma^{r-l_{j}} e^{-\delta|k|\left(r-l_{j}\right)} \\
& \times\left[\Gamma^{l_{j}} e^{-\delta|k| l_{j}} \omega\left(D^{l_{j}} f, K \Gamma \eta\right)+\Gamma^{l_{j}+1} e^{-\delta|k|\left(l_{j}+1\right)}\|f\|_{\left.C^{l_{j}} \eta\right]}\right. \\
\leq & K \ell^{2} \Gamma^{r-1} \frac{e^{(r+1) \delta}}{\sqrt{\tanh \delta}}\left(e^{[2 \varepsilon-(r-1) \delta]|k+1|} \omega\left(D^{r} f, K \Gamma \eta\right)+\Gamma e^{[2 \varepsilon-r \delta]|k+1|}\|f\|_{C^{r}} \eta\right) .
\end{aligned}
$$

Then, if $\delta>2 \varepsilon /(r-1), \omega\left(D^{r} f_{k}, \eta\right)$ is bounded independently of $k$, and, if $r \geq 2$, can be made small by taking $\Gamma$ small. 
Definition 2.2. Let $B_{2}$ be the ball around the origin in $\mathbb{R}^{d}$ of radius 2. Given a sequence $\mathcal{F}=\left\{f_{n}\right\}_{n \in \mathbb{Z}}, f_{n} \in C^{r}\left(B_{2}, \mathbb{R}^{d}\right)$ satisfying $f_{n}(0)=0$, we denote

$$
\begin{aligned}
A_{n} & =D f_{n}(0), \\
\tilde{f}_{n} & =f_{n}-A_{n}, \\
\tilde{\mathcal{F}} & =\left\{\tilde{f}_{n}\right\}_{n \in \mathbb{Z}}, \\
\|\mathcal{F}\|_{C^{r}\left(B_{2}\right)} & =\sup _{n \in \mathbb{Z}}\left\|f_{n}\right\|_{C^{r}\left(B_{2}\right)} .
\end{aligned}
$$

Remark 2.3. Given a family of $C^{r}$ maps, $\mathcal{F}=\left\{f_{n}\right\}_{n \in \mathbb{Z}}$ with $f_{n}(0)=0$, we will consider the family $\mathcal{F}_{\lambda}=\left\{f_{n, \lambda}\right\}_{n \in \mathbb{Z}}$, with $\lambda>0$ and

$$
f_{n, \lambda}(x)=\lambda^{-1} f_{n}(\lambda x) \text {. }
$$

Note that if $r \geq 2$ and $\|\mathcal{F}\|_{C^{r}\left(B_{2}\right)}<\infty$, then $\left\|\tilde{\mathcal{F}}_{\lambda}\right\|_{C^{r}\left(B_{2}\right)}$ can be assumed to be arbitrarily small by taking $\lambda$ small enough. Indeed, if $\mathcal{F}=\left\{f_{n}\right\}_{n \in \mathbb{Z}}$, it is clear that

$$
\begin{aligned}
\left|\tilde{f}_{n, \lambda}(x)\right| & =\lambda^{-1}\left|f_{n}(\lambda x)-D f_{n}(0) \lambda x\right| \\
& \leq \lambda\left\|D^{2} f_{n} \circ \lambda\right\|_{C^{0}\left(B_{2}\right)} \leq \lambda\|\mathcal{F}\|_{C^{r}\left(B_{2}\right)},
\end{aligned}
$$

and that

$$
\begin{aligned}
\left|D \tilde{f}_{n, \lambda}(x)\right| & =\left|D f_{n}(\lambda x)-D f_{n}(0)\right| \\
& \leq \lambda\left\|D^{2} f_{n} \circ \lambda\right\|_{C^{0}\left(B_{2}\right)} \leq \lambda\|\mathcal{F}\|_{C^{r}\left(B_{2}\right)} .
\end{aligned}
$$

For $2 \leq i \leq r$, one has

$$
\left|D^{i} \tilde{f}_{n, \lambda}(x)\right|=\lambda^{i-1}\left|D^{i} f_{n}(\lambda x)\right| \leq \lambda^{i-1}\|\mathcal{F}\|_{C^{r}\left(B_{2}\right)} .
$$

Moreover if $\left\{\right.$ graph $\left.V_{n}\right\}$ is a sequence of manifolds such that $V_{n}(0)=0$ and $f_{n}$ (graph $\left.\left(V_{n}\right)\right) \subset$ graph $\left(V_{n+1}\right)$, then, defining $V_{n, \lambda}(x):=\lambda^{-1} V_{n}(\lambda x)$, we have that graph $V_{n, \lambda}$ is a sequence of manifolds such that $f_{n, \lambda}\left(\operatorname{graph}\left(V_{n, \lambda}\right)\right) \subset$ graph $\left(V_{n+1, \lambda}\right)$.

Remark 2.4. If $r=1+\alpha, 0<\alpha<1$, and $D f_{n}$ are uniformly Hölder, that is, there exists $K>0$ independent of $n$ such that $\left|D f_{n}(x)-D f_{n}(y)\right| \leq K|x-y|^{\alpha}$, then $\|\tilde{\mathcal{F}}\|_{C^{1+\alpha}\left(B_{2}\right)}$ can also be assumed to be small taking $\lambda$ small. This can be easily checked since

$$
\begin{gathered}
\left|\tilde{f}_{n, \lambda}(x)\right|=\left|\int_{0}^{1}\left(D f_{n}(t \lambda x)-D f_{n}(0)\right) x d t\right| \leq K \lambda^{\alpha} /(\alpha+1), \\
\left|D \tilde{f}_{n, \lambda}(x)\right| \leq K \lambda^{\alpha}
\end{gathered}
$$

and

$$
\left|D \tilde{f}_{n, \lambda}(x)-D \tilde{f}_{n, \lambda}(y)\right|=\left|D f_{n}(\lambda x)-D f_{n}(\lambda y)\right| \leq K \lambda^{\alpha}|x-y|^{\alpha} .
$$

However, if $r=1$, it may not be possible to make $\left\|\tilde{\mathcal{F}}_{\lambda}\right\|_{C^{1}\left(B_{2}\right)}$ small. As an example, consider the family $\left\{f_{n}\right\}_{n \geq 1}$, where

$$
f_{n}(x)=\frac{\sin (n x)}{n} .
$$

It is clear that $\sup _{n}\left\|f_{n}\right\|_{C^{1}}=1$. But, for all $\lambda>0$,

$$
\tilde{f}_{n, \lambda}(x)=\lambda^{-1}\left(\frac{\sin (\lambda n x)}{n}-\lambda x\right) \rightarrow-x \quad \text { when } n \rightarrow \infty,
$$

which implies that $\left\|\tilde{\mathcal{F}}_{\lambda}\right\|_{C^{1}} \geq 1$, for all $\lambda>0$. 
As a consequence of Lemma 2.1. Theorem 1.9 will be immediately implied by the following theorem.

Theorem 2.5. Let $\mathcal{F}=\left\{f_{n}\right\}_{n \in \mathbb{Z}}$ be a family of $C^{r}$ maps in $\mathbb{R}^{q}, r \in \mathbb{N} \cup\{\infty, \omega\}$, $r \geq 2$. Assume that there is a decomposition $\mathbb{R}^{q}=E^{1} \oplus \cdots \oplus E^{p}$ invariant under $A_{n}$, that is, $A_{n} E^{i}=E^{i}$ and a set of real numbers $\tilde{\mu}_{1} \leq \tilde{\lambda}_{1}<\tilde{\mu}_{2} \leq \cdots \leq \tilde{\lambda}_{p-1}<\tilde{\mu}_{p} \leq \tilde{\lambda}_{p}$, such that

$$
v \in E^{i} \Rightarrow e^{\tilde{\mu}_{i}}|v| \leq\left|A_{n} v\right| \leq e^{\tilde{\lambda}_{i}}|v| \quad \text { for all } n \in \mathbb{Z} .
$$

Denote $I_{i}=\left[\tilde{\mu}_{i}, \tilde{\lambda}_{i}\right]$. Let $S \subset\{1, \ldots, p\}$ be such that $\left\{I_{i}: i \in S\right\}$ satisfies (6) and (7). Denote $E^{S}=\bigoplus_{i \in S} E^{i}, E^{S^{c}}=\bigoplus_{i \in S^{c}} E^{i}$. Assume that $\|\tilde{\mathcal{F}}\|_{C^{N_{S}+1}\left(B_{2}\right)}$ is sufficiently small, the modulus of continuity of $D^{r} \tilde{f}_{n}$ is uniformly bounded with respect to $n$ and $r \geq N_{S}+1$. Let $B_{1}$ be the unit ball in $E^{S}$. Then, there exist $C^{r}$ maps $V_{n}: B_{1} \subset E^{S} \rightarrow E^{S^{c}}$ in such a way that

(a) $\sup _{n \in \mathbb{Z}}\left\|V_{n}\right\|_{C^{r}\left(B_{1}\right)}<\infty$.

(b) $V_{n}(0)=0$ and $f_{n}\left(\operatorname{graph}\left(V_{n}\right)\right) \subset \operatorname{graph}\left(V_{n+1}\right)$.

(c) $D V_{n}(0)=0$.

Moreover, if $V_{n}$ and $\hat{V}_{n}$ are families of maps satisfying (b), we have that

(d) If

$$
\sup _{n \in \mathbb{Z}}\left\|V_{n}\right\|_{C^{m}\left(B_{1}\right)}<\infty, \quad \sup _{n \in \mathbb{Z}}\left\|\hat{V}_{n}\right\|_{C^{m}\left(B_{1}\right)}<\infty
$$

for some $m \leq r$, then $D^{i} V_{n}(0)=D^{i} \hat{V}_{n}(0)$ for all $i \leq m$.

(e) If $\sup _{n \in \mathbb{Z}}\left\|V_{n}\right\|_{C^{N_{S}+1}\left(B_{1}\right)} \leq 1$ and $\sup _{n \in \mathbb{Z}}\left\|\hat{V}_{n}\right\|_{C^{N_{S}+1}\left(B_{1}\right)} \leq 1$, then $V_{n}=$ $\hat{V}_{n}$. In particular, if $\hat{V}_{n}$ satisfies (b) and $\sup _{n \in \mathbb{Z}}\left\|\hat{V}_{n}\right\|_{C^{N_{S}+1}\left(B_{1}\right)} \leq 1, \hat{V}_{n}$ has to agree with the $V_{n}$ produced by this theorem and hence $\hat{V}$ is $C^{r}$ and $\sup _{n \in \mathbb{Z}}\left\|\hat{V}_{n}\right\|_{C^{r}\left(B_{1}\right)}<\infty$.

(f) If $\sup _{n \in \mathbb{Z}}\left\|V_{n}\right\|_{C^{N_{S}\left(B_{1}\right)}}<\infty$ and $V_{0} \in C^{k}\left(B_{1}\right)$ for some $N_{S}<k \leq r$, then $\sup _{n \in \mathbb{N}}\left\|V_{-n}\right\|_{C^{k}\left(B_{1}\right)}<\infty$.

Remark 2.6. If we assume that $\sup _{n \in \mathbb{Z}}\left\|V_{n}\right\|_{C^{N_{S}+1}\left(B_{1}\right)}$ and $\sup _{n \in \mathbb{Z}}\left\|\hat{V}_{n}\right\|_{C^{N_{S}+1}\left(B_{1}\right)}$ are just bounded in (e), scaling the maps we get the suprema to be smaller than 1 . This scaling implies working in a smaller domain and hence we obtain uniqueness in this smaller domain.

To apply Theorem 2.5 in proving Theorem 1.9, we take $\tilde{\lambda}_{i}=\lambda_{i}+2 \delta, \tilde{\mu}_{i}=\mu_{i}-2 \delta$, where $\delta$ is the small number we had to use in the estimates (iii) in Lemma 2.1. Recall that it is enough to take $\delta>2 \varepsilon$.

Note that if a set of intervals is non-resonant and contractive, and if we enlarge them by a sufficiently small quantity, then the enlarged intervals also satisfy the same properties. Hence, when applying Lemma 2.1 to the original situation, we have to pay attention to ensuring that $\delta$ is small enough - and thus $\varepsilon$ small enough - so that the non-resonance conditions and contractivity are still satisfied by the enlarged intervals. Then, taking $w_{n}(y)=\Phi_{n}\left(y, V_{n}(y)\right)$, all the conclusions follow.

The rest of the section is devoted to the proof of Theorem 2.5.

We denote by $A_{n}^{S}$ and $A_{n}^{S^{c}}$ the restrictions of $A_{n}$ to $E^{S}$ and $E^{S^{c}}$, respectively, and by $\Pi^{S}$ and $\Pi^{S^{c}}$ the projections onto $E^{S}$ and $E^{S^{c}}$, respectively. Note that in this setting they are independent of $n$. The invariance conclusion in (b) of Theorem 2.5 
can be expressed more explicitly as

$$
V_{n+1}\left(A_{n}^{S} x+\Pi^{S} \tilde{f}_{n}\left(x, V_{n}(x)\right)\right)=A_{n}^{S^{c}} V_{n}(x)+\Pi^{S^{c}} \tilde{f}_{n}\left(x, V_{n}(x)\right) .
$$

2.2. Obtaining a polynomial approximation. To show that such a sequence of maps $V_{n}$ satisfying (16) exists and verifies the uniqueness statements, we will start by determining candidates for their derivatives at zero.

If a sequence of differentiable functions $\left\{V_{n}\right\}_{n \in \mathbb{Z}}$ satisfies (16), we will show that the derivatives at the origin have to verify a certain functional equation (see (17) below). By studying this functional equation, we will show that, under the nonresonance conditions, there is one and only one bounded solution. Hence, if there is a sequence of differentiable maps, there is only one possibility for their jets at the origin. We will denote the solution of (17) (which is the only possibility for derivatives of the solutions of (16)) by $V_{n}^{0}$.

Incidentally, the analysis of (17) shows that if the non-resonance conditions are not met, it is possible that the equations for the jet do not have any solution. Hence, a fortiori, that there are no smooth invariant manifolds satisfying the conclusions of Theorem 1.9.

In Section 2.3 we will use $V_{n}^{0}$ to transform (16) into another equation (see (24) below) which has better contraction properties. This equation will be shown to have solutions using a contraction mapping argument. By re-examining the whole process, we will show that, indeed, the solution is a solution of the original problem and that, indeed, its jet is $V_{n}^{0}$.

We now proceed with the first step of deriving an equation for the jets at the origin. Taking formally $i$ derivatives of (16) at zero we obtain that, if the derivatives exist, they must satisfy

$$
D^{i} V_{n+1}(0)\left(A_{n}^{S}\right)^{\otimes i}=A_{n}^{S^{c}} D^{i} V_{n}(0)+R_{n, i}, \quad n \in \mathbb{Z},
$$

where $R_{n, i}$ is an expression that involves only derivatives of $V_{n}$ up to order $i-1$ and derivatives of $f_{n}$ up to order $i$. In order to solve (17), we recall that $D^{i} V_{n}(0)$ is a linear map from $\left(E^{S}\right)^{\otimes i}$ to $E^{S^{c}}$.

We claim that the hierarchy of equations (17) can be solved recursively and that the solution is unique.

Lemma 2.7. Let $\mathcal{F}=\left\{f_{n}\right\}_{n \in \mathbb{Z}}$ be a family of maps, $f_{n}: B_{2} \subset \mathbb{R}^{d} \rightarrow \mathbb{R}^{d}$, where $B_{2}$ is the ball centered at zero of radius 2 . Assume that $\|\mathcal{F}\|_{C^{k}\left(B_{2}\right)}<\infty$ and that $D f_{n}(0)=A_{n}$ satisfies the hypotheses of Theorem 2.5. Then, for all $1 \leq i \leq k$, the family of equations (17) has a unique solution in the Banach space

$$
\left\{\mathcal{T}^{i}=\left(T_{n}^{i}\right): T_{n}^{i} \in L\left(\left(E^{S}\right)^{\otimes i}, E^{S^{c}}\right),\left\|\mathcal{T}^{i}\right\|=\sup _{n \in \mathbb{Z}}\left\|T_{n}^{i}\right\|<\infty\right\} .
$$

Moreover, the norm of the solution can be made as small as we want by taking $\|\tilde{\mathcal{F}}\|_{C^{k}\left(B_{2}\right)}$ small.

For $i=1$ the only bounded solution is $D V_{n}(0)=0$.

This result proves parts (c) and (d) of Theorem 2.5.

Proof of the lemma. We will solve equations (17) in the components corresponding to the decomposition $\mathbb{R}^{d}=\bigoplus_{i=1}^{p} E^{i}$. Note that the decomposition

$$
\left(E^{S}\right)^{\otimes i}=\bigoplus_{j_{1}, \ldots, j_{i} \in S} E^{j_{1}} \otimes \cdots \otimes E^{j_{i}}
$$


is preserved by the linear map

$$
\left(A_{n}^{S}\right)^{\otimes i}=\bigoplus_{j_{1}, \ldots, j_{i} \in S} A_{n}^{j_{1}} \otimes \cdots \otimes A_{n}^{j_{i}}:\left(E^{S}\right)^{\otimes i} \rightarrow\left(E^{S}\right)^{\otimes i},
$$

where

$$
A_{n}^{j_{1}} \otimes \cdots \otimes A_{n}^{j_{i}}: E^{j_{1}} \otimes \cdots \otimes E^{j_{i}} \rightarrow E^{j_{1}} \otimes \cdots \otimes E^{j_{i}}
$$

and $\left\|A_{n}^{j_{1}} \otimes \cdots \otimes A_{n}^{j_{i}}\right\|=\left\|A_{n}^{j_{1}}\right\| \cdots\left\|A_{n}^{j_{i}}\right\|$.

If $T_{n}^{i} \in L\left(\left(E^{S}\right)^{\otimes i}, E^{S^{c}}\right)$, we can use the decomposition of $\left(E^{S}\right)^{\otimes i}$ and $E^{S^{c}}=$ $\bigoplus_{i \in S^{c}} E^{i}$ to write

where

$$
T_{n}^{i}=\bigoplus_{l \in S^{c}, j_{1}, \ldots, j_{i} \in S} \tau_{n, j_{1}, \ldots, j_{i}}^{l}
$$

$$
\tau_{n, j_{1}, \ldots, j_{i}}^{l}: E^{j_{1}} \otimes \cdots \otimes E^{j_{i}} \rightarrow E^{l}
$$

is linear. The same decomposition is made to the terms $R_{n, i}$ of (17), which also belong to $L\left(\left(E^{S}\right)^{\otimes i}, E^{S^{c}}\right)$, to obtain $R_{n, i}=\bigoplus_{l \in S^{c}, j_{1}, \ldots, j_{i} \in S} R_{n, j_{1}, \ldots, j_{i}}^{l}$.

Then, equations (17) are equivalent to the set of equations

$$
\tau_{n+1, j_{1}, \ldots, j_{i}}^{l} A_{n}^{j_{1}} \otimes \cdots \otimes A_{n}^{j_{i}}=A_{n}^{l} \tau_{n, j_{1}, \ldots, j_{i}}^{l}+R_{n, j_{1}, \ldots, j_{i}}^{l},
$$

with $n \in \mathbb{Z}, j_{1}, \ldots, j_{i} \in S$ and $l \in S^{c}$.

Equations (18) can be rewritten in either the form

$$
\tau_{n, j_{1}, \ldots, j_{i}}^{l}=\left(A_{n}^{l}\right)^{-1} \tau_{n+1, j_{1}, \ldots, j_{i}}^{l} A_{n}^{j_{1}} \otimes \cdots \otimes A_{n}^{j_{i}}-\left(A_{n}^{l}\right)^{-1} R_{n, j_{1}, \ldots, j_{i}}^{l}
$$

or the form

(20) $\tau_{n+1, j_{1}, \ldots, j_{i}}^{l}=A_{n}^{l} \tau_{n, j_{1}, \ldots, j_{i}}^{l}\left(A_{n}^{j_{1}} \otimes \cdots \otimes A_{n}^{j_{i}}\right)^{-1}+R_{n, j_{1}, \ldots, j_{i}}^{l}\left(A_{n}^{j_{1}} \otimes \cdots \otimes A_{n}^{j_{i}}\right)^{-1}$.

Equations (19) and (20) can be considered as fixed point equations, and the right-hand side of both of them defines continuous affine maps on the Banach space

$$
\begin{aligned}
\left\{\tau_{j_{1}, \ldots, j_{i}}^{l}=\left(\tau_{n, j_{1}, \ldots, j_{i}}^{l}\right)_{n \in \mathbb{Z}}:\right. & \tau_{n, j_{1}, \ldots, j_{i}}^{l} \in L\left(E^{j_{1}} \otimes \cdots \otimes E^{j_{i}}, E^{l}\right), \\
& \left.\left\|\tau_{j_{1}, \ldots, j_{i}}^{l}\right\|=\sup _{n \in \mathbb{Z}}\left\|\tau_{n, j_{1}, \ldots, j_{i}}^{l}\right\|<\infty\right\} .
\end{aligned}
$$

The map associated to (19) has a Lipschitz constant which is bounded from above by

$$
\exp \left(\tilde{\lambda}_{j_{1}}+\cdots+\tilde{\lambda}_{j_{i}}-\tilde{\mu}_{l}\right)
$$

while the one associated to (20) has a Lipschitz constant which is bounded from above by

$$
\exp \left(\tilde{\lambda}_{l}-\tilde{\mu}_{j_{1}}-\cdots-\tilde{\mu}_{j_{i}}\right) .
$$

Since the intervals $\left\{I_{i}\right\}_{i \in S}$ are non-resonant, one of the two numbers (21) or (22) is smaller than 1. Then, one of the two equations (19) or (20) has a right-hand side which defines a contraction. Hence, one of (19) or (20) can be solved by the contraction mapping principle. Since both equations (19) and (20) are equivalent to (18), we have proved that the equations (18) have a unique solution.

All terms $R_{n, i}$ have a factor $D^{j} \tilde{f}(0,0), 2 \leq j \leq i$, which is small if $\|\tilde{\mathcal{F}}\|_{C^{k}\left(B_{2}\right)}$ is small. Therefore, $R_{n, j_{1}, \ldots, j_{i}}$ are also small and hence the non-homogeneous parts of (19) and (20) are small. From that we deduce that the unique solutions of equations (18) are small. 
Note that the equations for the first derivative are homogeneous. It follows, then, that $D V_{n}(0)=0$ is the unique bounded solution of equations (17) for $i=1$. This establishes Lemma 2.7.

2.3. The high order part of the manifolds. We now rewrite the invariance equations in such a way that we can take advantage of the fact that we already know the low order terms of the expansion of its solution.

We will write the sequence of maps $\mathcal{V}=\left\{V_{n}\right\}_{n \in \mathbb{Z}}$ in (16) in the form $\mathcal{V}=\mathcal{V}^{0}+\mathcal{U}$ with $\mathcal{V}^{0}=\left\{V_{n}^{0}\right\}_{n \in \mathbb{Z}}, \mathcal{U}=\left\{U_{n}\right\}_{n \in \mathbb{Z}}$ and

$$
V_{n}=V_{n}^{0}+U_{n}
$$

where $V_{n}^{0}$ is the unique polynomial of degree $N_{S}$ whose derivatives at 0 satisfy equations (17) and $D^{i} U_{n}(0)=0$, for $0 \leq i \leq N_{S}$. Note that $\left\|\mathcal{V}^{0}\right\|_{C^{r}}$ can be made as small as we need.

Next we reformulate our original problem in terms of the $U_{n}$. As it turns out, this will be very convenient since we will have that the $U_{n}$ are maps that vanish to high order. For such maps, composing with contractions on the right leads to a rather strong contraction factor. If we substitute (23) in the invariance equation (16) we obtain a fixed point equation $\mathcal{U}=\sigma(\mathcal{U})$, for a sequence of maps $\mathcal{U}=\left\{U_{n}\right\}_{n \in \mathbb{Z}}$, where the operator $\sigma$ is defined by

$$
\begin{aligned}
\sigma(\mathcal{U})_{n}(x)=\left(A_{n}^{S^{c}}\right)^{-1}[ & \left(V_{n+1}^{0}+U_{n+1}\right) \circ \psi(\mathcal{U})_{n}(x) \\
& \left.-\Pi^{S^{c}} \tilde{f}_{n}\left(x, V_{n}^{0}(x)+U_{n}(x)\right)\right]-V_{n}^{0}(x)
\end{aligned}
$$

with

$$
\psi(\mathcal{U})_{n}(x)=A_{n}^{S} x+\Pi^{S} \tilde{f}_{n}\left(x, V_{n}^{0}(x)+U_{n}(x)\right)
$$

A simple algebraic manipulation shows that, at least formally, ignoring questions of whether the compositions can be defined is equivalent to saying that $\mathcal{U}$ is a fixed point of (24) rather than saying that $\mathcal{V}^{0}+\mathcal{U}$ is a solution of (16). The plan will be to produce fixed points of (24) in a space of well-behaved functions for which the algebraic manipulations involved in transforming (16) into (24) can be justified. For the moment, we will consider only the case that $r \in \mathbb{N}$. Later we will see how this result can be extended to the cases $r=\omega, \infty$.

More precisely, we consider $\sigma$ acting on the following spaces:

$$
\begin{gathered}
\mathcal{X}_{k}=\left\{\mathcal{U}=\left\{U_{n}\right\}_{n \in \mathbb{Z}}: U_{n} \in C^{k}\left(B_{1}\right), D^{i} U_{n}(0)=0,0 \leq i \leq N_{S},\right. \\
\left.\|\mathcal{U}\|_{\mathcal{X}_{k}}:=\sup _{n \in \mathbb{Z}} \max _{N_{S}<i \leq k}\left\|D^{i} U_{n}\right\|_{C^{0}}<\infty\right\},
\end{gathered}
$$

and

$$
\begin{gathered}
\mathcal{X}^{0}=\left\{\mathcal{U}=\left\{U_{n}\right\}_{n \in \mathbb{Z}}: U_{n} \in C^{N_{S}}\left(B_{1}\right), D^{i} U_{n}(0)=0,0 \leq i \leq N_{S},\right. \\
\left.\|\mathcal{U}\|_{\mathcal{X}^{0}}:=\sup _{n \in \mathbb{Z}} \sup _{x \in B_{1} \backslash\{0\}}|x|^{-1}\left|D^{N_{S}} U_{n}(x)\right|<\infty\right\} .
\end{gathered}
$$

By the mean value theorem, if $k>N_{S}, \mathcal{X}_{k} \subset \mathcal{X}^{0}$. Moreover, if $\mathcal{U} \in \mathcal{X}_{k},\|\mathcal{U}\|_{\mathcal{X}^{0}} \leq$ $\|\mathcal{U}\| \mathcal{X}_{k}$. 
Let $\mathcal{B}_{N_{S}+1}=\left\{\mathcal{U} \in \mathcal{X}_{N_{S}+1}:\|\mathcal{U}\|_{\mathcal{X}_{N_{S}+1}} \leq 1\right\}$ and $\mathcal{B}\left(\rho_{N_{S}+2}, \ldots, \rho_{k}\right)$ be the set of families $\mathcal{U}=\left\{U_{n}\right\}_{n \in \mathbb{Z}} \in \mathcal{X}_{k}$ such that

$$
\begin{aligned}
& \sup _{n \in \mathbb{Z}}\left\|D^{N_{S}+1} U_{n}\right\|_{C^{0}} \leq 1, \\
& \sup _{n \in \mathbb{Z}}\left\|D^{j} U_{n}\right\|_{C^{0}} \leq \rho_{j}, \quad N_{S}+1<j \leq k .
\end{aligned}
$$

Note that the last condition is void if $k=N_{S}+1$. Clearly $\mathcal{B}\left(\rho_{N_{S}+2}, \ldots, \rho_{k}\right) \subset$ $\mathcal{B}_{N_{S}+1}$. We remark that if $\mathcal{U} \in \mathcal{X}_{k}$, we can reconstruct $U_{n}$ from $D^{N_{S}+1} U_{n}$ and $\left\|D^{i} U_{n}(x)\right\| /|x|^{N_{S}+1-i} \leq\left(1 /\left(N_{S}+1-i\right) !\right)\left\|U_{n}\right\|_{C^{N_{S}+1}}$, for $0 \leq i \leq N_{S}+1$. Therefore we get that if $\left\|D^{N_{S}+1}(\sigma \mathcal{U})_{n}\right\|_{C^{0}} \leq 1$, we also have that $\left\|D^{i}(\sigma \mathcal{U})_{n}\right\|_{C^{0}} \leq 1$, for $0 \leq i \leq N_{S}$.

Lemma 2.8. Let $\sigma$ be defined as in (24). Under appropriate smallness conditions on $\|\tilde{\mathcal{F}}\|_{C^{N_{S}+1}\left(B_{2}\right)}$ :

(i) $\sigma\left(\mathcal{B}_{N_{S}+1}\right) \subset \mathcal{B}_{N_{S}+1}$

(ii) $\sigma$ restricted to $\mathcal{B}_{N_{S}+1}$ is a contraction in the $\mathcal{X}^{0}$-norm.

Moreover, under the same smallness conditions on $\|\tilde{\mathcal{F}}\|_{C^{N_{S}+1}\left(B_{2}\right)}$ needed for (i) and (ii) there exist numbers $\rho_{N_{S}+2}, \ldots, \rho_{k}>0$ such that

(iii) $\sigma\left(\mathcal{B}\left(\rho_{N_{S}+2}, \ldots, \rho_{k}\right)\right) \subset \mathcal{B}\left(\rho_{N_{S}+2}, \ldots, \rho_{k}\right)$ for $N_{S}+2 \leq k \leq r$.

For future reference, it will be important to note that the smallness conditions assumed in Lemma 2.8 are only smallness assumptions on $\|\tilde{\mathcal{F}}\|_{C^{N_{S}+1}\left(B_{2}\right)}$ and do not change as we increase $r$.

Before proving the lemma we first establish some bounds for $\psi$. Given $\mathcal{U}, \hat{\mathcal{U}} \in$ $\mathcal{B}_{N_{S}+1}$ we have

$$
\begin{aligned}
\left\|D \psi(\mathcal{U})_{n}\right\| & \leq\left\|A_{n}^{S}\right\|+K\|\tilde{\mathcal{F}}\|_{C^{1}} \\
|x|^{-1}\left\|\psi(\mathcal{U})_{n}(x)\right\| & \leq\left\|A_{n}^{S}\right\|+K\|\tilde{\mathcal{F}}\|_{C^{1}} \\
|x|^{-1}\left\|\left(\psi(\mathcal{U})_{n}-\psi(\hat{\mathcal{U}})_{n}\right)(x)\right\| & \leq K\|\tilde{\mathcal{F}}\|_{C^{1}}\|\mathcal{U}-\hat{\mathcal{U}}\|_{\mathcal{X}^{0}} \\
|x|^{-1}\left\|D\left(\psi(\mathcal{U})_{n}-\psi(\hat{\mathcal{U}})_{n}\right)(x)\right\| & \leq K\|\tilde{\mathcal{F}}\|_{C^{2}}\|\mathcal{U}-\hat{\mathcal{U}}\|_{\mathcal{X}^{0}}
\end{aligned}
$$

where $K$ is a constant independent of $n$. Formula (28) is straightforward from the definition of $\psi$ in (25), while (29)-(31) follow from the fact that $\tilde{f}_{n}(0)=0$, $D \tilde{f}_{n}(0)=0, V_{n}^{0}(0)=0$ and the derivatives of $V_{n}^{0}$ and $U_{n}$ are uniformly bounded.

Proof of Lemma 2.8. It is clear that, by the choice of the polynomials $V_{n}^{0}$,

$$
D^{i} \sigma(\mathcal{U})_{n}(0)=0, \quad 0 \leq i \leq N_{S} .
$$

We observe that, for $k \geq 2$, by the Faa-di Bruno formula,

$$
D^{k} \sigma(\mathcal{U})_{n}=\left(A_{n}^{S^{c}}\right)^{-1} D^{k} U_{n+1} \circ \psi(\mathcal{U})_{n} D \psi(\mathcal{U})_{n}^{\otimes k}+B_{n}(\mathcal{U}) D^{k} U_{n}+R_{n, k}(\mathcal{U}),
$$

where

$$
\begin{aligned}
B_{n}(\mathcal{U})= & \left(A_{n}^{S^{c}}\right)^{-1} D\left(V_{n+1}^{0}+U_{n+1}\right) \circ \psi(\mathcal{U})_{n} \Pi^{S} D_{2} \tilde{f}_{n} \circ \eta(\mathcal{U})_{n} \\
& -\left(A_{n}^{S^{c}}\right)^{-1} \Pi^{S^{c}} D_{2} \tilde{f}_{n} \circ \eta(\mathcal{U})_{n}
\end{aligned}
$$

is linear,

$$
\eta(\mathcal{U})_{n}=\left(\mathrm{Id}, V_{n}^{0}+U_{n}\right)
$$


and since $k \geq N_{S}+1$,

$$
\begin{aligned}
R_{n, k}(\mathcal{U})= & \left(A_{n}^{S^{c}}\right)^{-1}\left(\sum_{l=2}^{N_{S}} \sum_{*} c_{j_{1}, \ldots, j_{l}}^{k, l} D^{l} V_{n+1}^{0} \circ \psi(\mathcal{U})_{n} D^{j_{1}} \psi(\mathcal{U})_{n} \otimes \cdots \otimes D^{j_{l}} \psi(\mathcal{U})_{n}\right. \\
& +\sum_{l=2}^{k-1} \sum_{*} c_{j_{1}, \ldots, j_{l}}^{k, l} D^{l} U_{n+1} \circ \psi(\mathcal{U})_{n} D^{j_{1}} \psi(\mathcal{U})_{n} \otimes \cdots \otimes D^{j_{l}} \psi(\mathcal{U})_{n} \\
& +D\left(V_{n+1}^{0}+U_{n+1} \circ \psi \psi(\mathcal{U})_{n}\right. \\
& \times \Pi^{S} \sum_{l=2}^{k} \sum_{*} c_{j_{1}, \ldots, j_{l}}^{k, l} D^{l} \tilde{f}_{n} \circ \eta(\mathcal{U})_{n} D^{j_{1}} \eta(\mathcal{U})_{n} \otimes \cdots \otimes D^{j_{l}} \eta(\mathcal{U})_{n} \\
& \left.-\Pi^{S^{c}} \sum_{l=2}^{k} \sum_{*} c_{j_{1}, \ldots, j_{l}}^{k, l} D^{l} \tilde{f}_{n} \circ \eta(\mathcal{U})_{n} D^{j_{1}} \eta(\mathcal{U})_{n} \otimes \cdots \otimes D^{j_{l}} \eta(\mathcal{U})_{n}\right)
\end{aligned}
$$

where $\sum_{*}$ stands for the sum over the indices $j_{i}$ such that $1 \leq j_{1}, \ldots, j_{l} \leq k$ and $j_{1}+\cdots+j_{l}=k$ and the coefficients $c_{j_{1}, \ldots, j_{l}}^{k, l}$ are combinatorial numbers.

Note that a first derivative of $\tilde{f}_{n}$ appears as a factor in each term in $B_{n}(\mathcal{U})$. Also note that $R_{n, k}(\mathcal{U})$ consists of a finite sum of terms. Some of them have explicitly a derivative of $\tilde{f}_{n}$ as a factor. The other terms have a factor of the form

$$
D^{j_{1}} \psi(\mathcal{U})_{n} \otimes \cdots \otimes D^{j_{l}} \psi(\mathcal{U})_{n},
$$

where

$$
j_{1}+\cdots+j_{l}=k \quad \text { and } \quad l \leq k-1 .
$$

Because of (37), there is some $i$ such that $j_{i} \geq 2$ and therefore, among the factors in (36), there is a factor of the form

$$
D^{j_{i}} \psi(\mathcal{U})_{n}=D^{j_{i}}\left(\tilde{f}_{n} \circ \eta_{n}(\mathcal{U})\right) .
$$

Now we consider the case $k=N_{S}+1$. The previous factors can be made arbitrarily small by assuming that $\|\tilde{\mathcal{F}}\|_{C^{N_{S}+1}}$ is sufficiently small. As a consequence, for any given $\nu>0$, if $\|\tilde{\mathcal{F}}\|_{C^{N_{S}+1}}$ is small enough, we have that

$$
\left\|B_{n}(\mathcal{U})\right\|<\nu \quad \text { and } \quad\left\|R_{n, N_{S}+1}(\mathcal{U})\right\|_{C^{0}}<\nu .
$$

Since $\sup _{n}\left\|\left(A_{n}^{S^{c}}\right)^{-1}\right\|\left\|A_{n}^{S}\right\|^{N_{S}+1}<1$, there exists $\nu>0$ such that

$$
\gamma:=\left\|\left(A_{n}^{S^{c}}\right)^{-1}\right\|\left(\left\|A_{n}^{S}\right\|+\nu\right)^{N_{S}+1}+2 \nu<1
$$

and hence

$$
\left\|D^{N_{S}+1} \sigma(\mathcal{U})_{n}\right\|_{C^{0}} \leq \sup _{n \in \mathbb{Z}}\left(\left\|\left(A_{n}^{S^{c}}\right)^{-1}\right\|\left(\left\|A_{n}^{S}\right\|+\nu\right)^{N_{S}+1}+\nu\right)\left\|D^{N_{S}+1} \mathcal{U}\right\|_{C^{0}}+\nu \leq \gamma,
$$

for all $\mathcal{U}$ in $\mathcal{B}_{N_{S}+1}$. This proves (i).

If $N_{S}+1<k \leq r$, from (32) we have that

$$
\left\|D^{k} \sigma(\mathcal{U})_{n}\right\| \leq \gamma \sup _{n \in \mathbb{Z}}\left\|D^{k} U_{n}\right\|+Q\left(\rho_{N_{S}+2}, \ldots, \rho_{k-1}\right),
$$

where $Q$ is a polynomial. Therefore, since $\gamma<1$, there exists $\rho_{k}>0$ such that $\rho_{k}=\gamma \rho_{k}+Q\left(\rho_{N_{S}+2}, \ldots, \rho_{k-1}\right)$ and $\sigma\left(\mathcal{B}\left(\rho_{N_{S}+2}, \ldots, \rho_{k}\right)\right) \subset \mathcal{B}\left(\rho_{N_{S}+2}, \ldots, \rho_{k}\right)$. 
To prove (ii) we take derivatives, and we obtain

$$
\begin{aligned}
& |x|^{-1}\left\|D^{N_{S}}\left(\sigma(\mathcal{U})_{n}-\sigma(\hat{\mathcal{U}})_{n}\right)(x)\right\| \\
& \leq \quad|x|^{-1}\left\|\left(A_{n}^{S^{c}}\right)^{-1} D^{N_{S}}\left(U_{n+1}-\hat{U}_{n+1}\right) \circ \psi(\mathcal{U})_{n}(x) D \psi(\mathcal{U})_{n}(x)^{\otimes N_{S}}\right\| \\
& \quad+|x|^{-1} \|\left(A_{n}^{S^{c}}\right)^{-1}\left(D^{N_{S}} \hat{U}_{n+1} \circ \psi(\mathcal{U})_{n}(x)\right. \\
& \left.\quad-D^{N_{S}} \hat{U}_{n+1} \circ \psi(\hat{\mathcal{U}})_{n}\right)(x) D \psi(\mathcal{U})_{n}(x)^{\otimes N_{S}} \| \\
& \quad+|x|^{-1} \|\left(A_{n}^{S^{c}}\right)^{-1} D^{N_{S}} \hat{U}_{n+1} \circ \psi(\hat{\mathcal{U}})_{n}(x) \\
& \quad \times\left(D \psi(\mathcal{U})_{n}(x)^{\otimes N_{S}}-D \psi(\hat{\mathcal{U}})_{n}(x)^{\otimes N_{S}}\right) \| \\
& \quad+|x|^{-1}\left\|B_{n}(\mathcal{U})(x) D^{N_{S}}\left(U_{n}-\hat{U}_{n}\right)(x)\right\| \\
& \quad+|x|^{-1}\left\|\left(B_{n}(\mathcal{U})-B_{n}(\hat{\mathcal{U}})\right)(x) D^{N_{S}} \hat{U}_{n}(x)\right\| \\
& \quad+|x|^{-1}\left\|\left(R_{n, N_{S}}(\mathcal{U})-R_{n, N_{S}}(\hat{\mathcal{U}})\right)(x)\right\| .
\end{aligned}
$$

Taking into account inequalities (28) and (29), we bound (38) as follows:

$$
\begin{aligned}
& |x|^{-1}\left\|\left(A_{n}^{S^{c}}\right)^{-1} D^{N_{S}}\left(U_{n+1}-\hat{U}_{n+1}\right) \circ \psi(\mathcal{U})_{n}(x) D \psi(\mathcal{U})_{n}(x)^{\otimes N_{S}}\right\| \\
& \quad \leq|x|^{-1}\left|\psi(\mathcal{U})_{n}(x)\right|\left\|\left(A_{n}^{S^{c}}\right)^{-1}\right\|\left\|D \psi(\mathcal{U})_{n}\right\|^{N_{S}}\left\|U_{n+1}-\hat{U}_{n+1}\right\|_{\mathcal{X}^{0}} \\
& \quad \leq\left\|\left(A_{n}^{S^{c}}\right)^{-1}\right\|\left(\left\|A_{n}^{S}\right\|+K\|\tilde{\mathcal{F}}\|_{C^{1}}\right)^{N_{S}+1}\left\|U_{n+1}-\hat{U}_{n+1}\right\|_{\mathcal{X}^{0}} .
\end{aligned}
$$

To bound (39), we use inequality (30) and the fact that $\mathcal{U}, \hat{\mathcal{U}} \in \mathcal{B}_{N_{S}+1}$. In this way,

$$
\begin{aligned}
& |x|^{-1}\left\|\left(A_{n}^{S^{c}}\right)^{-1}\left(D^{N_{S}} \hat{U}_{n+1} \circ \psi(\mathcal{U})_{n}(x)-D^{N_{S}} \hat{U}_{n+1} \circ \psi(\hat{\mathcal{U}})_{n}(x)\right) D \psi(\mathcal{U})_{n}(x)^{\otimes N_{S}}\right\| \\
& \quad \leq\left\|\left(A_{n}^{S^{c}}\right)^{-1}\right\|\left\|D \psi(\mathcal{U})_{n}\right\|^{N_{S}}\|\hat{\mathcal{U}}\|_{C^{N_{S}+1}}|x|^{-1}\left\|\left(\psi(\mathcal{U})_{n}-\psi(\hat{\mathcal{U}})_{n}\right)(x)\right\| \\
& \quad \leq K\left\|\left(A_{n}^{S^{c}}\right)^{-1}\right\|\left(\left\|A_{n}^{S}\right\|+K\|\tilde{\mathcal{F}}\|_{C^{1}}\right)^{N_{S}}\|\hat{\mathcal{U}}\|_{C^{N_{S}}+1}\|\tilde{\mathcal{F}}\|_{C^{1}}\|\mathcal{U}-\hat{\mathcal{U}}\|_{\mathcal{X}^{0}} .
\end{aligned}
$$

Term (40) can be bounded in the following way, using inequalities (31) and (28):

$$
\begin{aligned}
& |x|^{-1}\left\|\left(A_{n}^{S^{c}}\right)^{-1} D^{N_{S}} \hat{U}_{n+1} \circ \psi(\hat{\mathcal{U}})_{n}(x)\left(D \psi(\mathcal{U})_{n}(x)^{\otimes N_{S}}-D \psi(\hat{\mathcal{U}})_{n}(x)^{\otimes N_{S}}\right)\right\| \\
& \leq \quad\left\|\left(A_{n}^{S^{c}}\right)^{-1}\right\|\left(\left\|A_{n}^{S}\right\|+K\|\tilde{\mathcal{F}}\|_{C^{1}}\right)\left\|\hat{U}_{n+1}\right\|_{C^{N_{S}}} \\
& \quad|x|^{-1}\left\|D \psi(\mathcal{U})_{n}(x)^{\otimes N_{S}}-D \psi(\hat{\mathcal{U}})_{n}(x)^{\otimes N_{S}}\right\| \\
& \leq \quad N_{S} K\left\|\left(A_{n}^{S^{c}}\right)^{-1}\right\|\left(\left\|A_{n}^{S}\right\|+K\|\tilde{\mathcal{F}}\|_{C^{1}}\right)^{N_{S}}\left\|\hat{U}_{n+1}\right\|_{C^{N_{S}}}\|\tilde{\mathcal{F}}\|_{C^{2}}\|\mathcal{U}-\hat{\mathcal{U}}\|_{\mathcal{X}^{0}}
\end{aligned}
$$

where we have bounded

$$
\begin{aligned}
& |x|^{-1}\left\|D \psi(\mathcal{U})_{n}(x)^{\otimes N_{S}}-D \psi(\hat{\mathcal{U}})_{n}(x)^{\otimes N_{S}}\right\| \\
& \quad \leq \sum_{j=1}^{N_{S}}|x|^{-1}\left\|D \psi(\mathcal{U})_{n}(x)-D \psi(\hat{\mathcal{U}})_{n}(x)\right\|\left\|D \psi(\mathcal{U})_{n}\right\|^{N_{S}-j}\left\|D \psi(\hat{\mathcal{U}})_{n}\right\|^{j-1} \\
& \quad \leq N_{S} K\left(\left\|A_{n}^{S}\right\|+K\|\tilde{\mathcal{F}}\|_{C^{1}}\right)^{N_{S}-1}\|\tilde{\mathcal{F}}\|_{C^{2}}\|\mathcal{U}-\hat{\mathcal{U}}\|_{\mathcal{X}^{0}} .
\end{aligned}
$$

Term (41) can be easily bounded taking into account that

$$
\left\|B_{n}(\mathcal{U})\right\| \leq \tilde{K}\|\tilde{\mathcal{F}}\|_{C^{1}}
$$

for some $\tilde{K}>0$.

To obtain a bound for (42) we proceed in the following way:

$$
\begin{aligned}
\left\|B_{n}(\mathcal{U})-B_{n}(\hat{\mathcal{U}})\right\| \leq & \left\|\left(A_{n}^{S^{c}}\right)^{-1}\right\| \| D\left(V_{n+1}^{0}+U_{n+1}\right) \circ \psi(\mathcal{U})_{n} \Pi^{S} D_{2} \tilde{f}_{n} \circ \eta(\mathcal{U})_{n} \\
& -D\left(V_{n+1}^{0}+\hat{U}_{n+1}\right) \circ \psi(\hat{\mathcal{U}})_{n} \Pi^{S} D_{2} \tilde{f}_{n} \circ \eta(\hat{\mathcal{U}})_{n} \| \\
+ & \left\|\Pi^{S^{c}} D_{2} \tilde{f}_{n} \circ \eta(\mathcal{U})_{n}-\Pi^{S^{c}} D_{2} \tilde{f}_{n} \circ \eta(\hat{\mathcal{U}})_{n}\right\| .
\end{aligned}
$$


Since $\left\|\eta(\mathcal{U})_{n}(x)-\eta(\hat{\mathcal{U}})_{n}(x)\right\|=\left\|U_{n}(x)-\hat{U}_{n}(x)\right\|$, the last term of (44) can be bounded immediately by

$$
\|\tilde{\mathcal{F}}\|_{C^{2}}\|\mathcal{U}-\hat{\mathcal{U}}\|_{\mathcal{X}^{0}}
$$

The first term in (44) can be split into the three following terms that can be easily bounded:

$$
\begin{gathered}
\left\|D\left(U_{n+1}-\hat{U}_{n+1}\right) \circ \psi(\mathcal{U})_{n} \Pi^{S} D_{2} \tilde{f}_{n} \circ \eta(\mathcal{U})_{n}\right\|, \\
\left\|\left(D\left(V_{n+1}^{0}+\hat{U}_{n+1}\right) \circ \psi(\mathcal{U})_{n}-D\left(V_{n+1}^{0}+\hat{U}_{n+1}\right) \circ \psi(\hat{\mathcal{U}})_{n}\right) \Pi^{S} D_{2} \tilde{f}_{n} \circ \eta(\mathcal{U})_{n}\right\|, \\
\left\|D\left(V_{n+1}^{0}+\hat{U}_{n+1}\right) \circ \psi(\hat{\mathcal{U}})_{n}\left(\Pi^{S} D_{2} \tilde{f}_{n} \circ \eta(\hat{\mathcal{U}})_{n}-\Pi^{S} D_{2} \tilde{f}_{n} \circ \eta(\mathcal{U})_{n}\right)\right\| .
\end{gathered}
$$

Finally, to bound (43), we recall that $R_{n, N_{S}}(\mathcal{U})$ is a finite sum of terms having expressions of the following forms:

(1) Terms of the form

$$
D^{l} V_{n+1}^{0} \circ \psi(\mathcal{U})_{n} D^{j_{1}} \psi(\mathcal{U})_{n} \otimes \cdots \otimes D^{j_{l}} \psi(\mathcal{U})_{n}, \quad 2 \leq l \leq N_{S}, j_{1}+\cdots+j_{l}=k .
$$

The norm of their difference is easily bounded by

$$
\begin{aligned}
& |x|^{-1}\left\|\left(D^{l} V_{n+1}^{0} \circ \psi(\mathcal{U})_{n}-D^{l} V_{n+1}^{0} \circ \psi(\hat{\mathcal{U}})_{n}\right) D^{j_{1}} \psi(\mathcal{U})_{n} \otimes \cdots \otimes D^{j_{l}} \psi(\mathcal{U})_{n}\right\| \\
& +|x|^{-1} \| D^{l} V_{n+1}^{0} \circ \psi(\hat{\mathcal{U}})_{n}\left(D^{j_{1}} \psi(\mathcal{U})_{n} \otimes \cdots \otimes D^{j_{l}} \psi(\mathcal{U})_{n}\right. \\
& \left.\quad-D^{j_{1}} \psi(\hat{\mathcal{U}})_{n} \otimes \cdots \otimes D^{j_{l}} \psi(\hat{\mathcal{U}})_{n}\right) \| .
\end{aligned}
$$

Both differences can be bounded by some constant times

$$
\|\tilde{\mathcal{F}}\|_{C^{2}}\|\mathcal{U}-\hat{\mathcal{U}}\|_{\mathcal{X}^{0}}
$$

(2) Terms of the form

$$
D^{l} U_{n+1} \circ \psi(\mathcal{U})_{n} D^{j_{1}} \psi(\mathcal{U})_{n} \otimes \cdots \otimes D^{j_{l}} \psi(\mathcal{U})_{n}, \quad 2 \leq l \leq N_{S}-1, j_{1}+\cdots+j_{l}=k .
$$

Since $\mathcal{U} \in \mathcal{B}_{N_{S}+1}$, their difference can be bounded as in the previous case.

(3) Terms of the form

$$
D^{l} \tilde{f}_{n} \circ \eta(\mathcal{U})_{n} D^{j_{1}} \eta(\mathcal{U})_{n} \otimes \cdots \otimes D^{j_{l}} \eta(\mathcal{U})_{n}, \quad 1 \leq l \leq N_{S}, j_{1}+\cdots+j_{l}=k,
$$

which can be treated as before, since $\tilde{f}_{n}$ is $C^{N_{S}+1}$.

(4) Terms of the form

$D\left(V_{n+1}^{0}+U_{n+1}\right) \circ \psi(\mathcal{U})_{n} \Pi^{S} D^{l} \tilde{f}_{n} \circ \eta(\mathcal{U})_{n} D^{j_{1}} \eta(\mathcal{U})_{n} \otimes \cdots \otimes D^{j_{l}} \eta(\mathcal{U})_{n}$,

where $2 \leq l \leq N_{S}, j_{1}+\cdots+j_{l}=k$, that can be bounded like the preceding ones.

Putting together the estimates for terms of the form (1)-(4), it follows that the Lipschitz constant of $\sigma$ is less than

$$
\left\|\left(A_{n}^{S^{c}}\right)^{-1}\right\|\left(\left\|A_{n}^{S}\right\|+\nu\right)^{N_{S}+1}+\nu,
$$

where $\nu$ is as small as we need taking $\|\tilde{\mathcal{F}}\|_{C^{N_{s}+1}}$ small enough. Then $\sigma$ is a contraction on $\mathcal{X}^{0}$.

Now we proceed to argue that the fixed point thus produced satisfies the claimed properties.

By (i) and (ii) it is clear that there is a fixed point of $\sigma$ in $\mathcal{X}^{0}$, hence, a solution of (16). Moreover, because of (i), this fixed point of $\sigma$ also belongs to the $\mathcal{X}^{0}$-closure of $\mathcal{B}\left(\rho_{N_{S}+2}, \ldots, \rho_{k}\right), N_{S}+1 \leq k \leq r$. 
By Lemma 2.8 and Proposition A2 in [LI73], we get the existence of a $C^{r-1+l i p}$ solution of the invariance equation. Furthermore, given any $\mathcal{U} \in \mathcal{B}\left(\rho_{N_{S}+2}, \ldots, \rho_{k}\right)$, the sequence $\sigma^{j}(\mathcal{U})$ tends in the $C^{r-1}$-norm to the fixed point of $\sigma$.

Now, to check that this solution is in fact $C^{r}$, we note first that

$$
\left\|R_{n, r}(\mathcal{U})-R_{n, r}(\hat{\mathcal{U}})\right\|_{C^{0}} \rightarrow 0
$$

when $\|\mathcal{U}-\hat{\mathcal{U}}\|_{C^{r-1}} \rightarrow 0$ and $\mathcal{U}, \hat{\mathcal{U}} \in \mathcal{B}\left(\rho_{N_{S}+2}, \ldots, \rho_{k}\right)$. This fact is trivial for all the terms involving derivatives up to order $r-1$ of $\tilde{\mathcal{F}}, \mathcal{U}$ and $\hat{\mathcal{U}}$, since these derivatives are in fact Lipschitz. The only terms involving $r$ derivatives are

$$
D\left(V_{n+1}^{0}+U_{n+1}\right) \circ \psi(\mathcal{U})_{n} \Pi^{S} D^{r} \tilde{f}_{n} \circ \eta(\mathcal{U})_{n} D \eta(\mathcal{U})_{n}^{\otimes r}
$$

and

$$
\Pi^{S^{c}} D^{r} \tilde{f}_{n} \circ \eta(\mathcal{U})_{n} D \eta(\mathcal{U})_{n}^{\otimes r}
$$

which are continuous in $\mathcal{U}$ since $D^{r} \tilde{f}_{n}$ and $\eta$ are continuous with modulus of continuity independent of $n$.

Next we consider the sequence of maps $\mathcal{U}^{l}=\left\{U_{n}^{l}\right\}_{n \in \mathbb{Z}}$ defined by

$$
U_{n}^{0}=0, \quad n \in \mathbb{Z}, \quad \mathcal{U}^{l+1}=\sigma\left(\mathcal{U}^{l}\right) .
$$

The preceding arguments show that the sequence $\mathcal{U}^{l} \in \mathcal{B}\left(\rho_{N_{S}+2}, \ldots, \rho_{k}\right)$ converges in the $C^{r-1}$-norm to some $\mathcal{U}^{\infty}=\left\{U_{n}^{\infty}\right\}_{n \in \mathbb{Z}}$. The sequence of $r$-derivatives satisfies the recurrence relation

$$
D^{r} U_{n}^{l+1}=\left(A_{n}^{S^{c}}\right)^{-1} D^{r} U_{n+1}^{l} \circ \psi\left(\mathcal{U}^{l}\right)_{n} D \psi\left(\mathcal{U}^{l}\right)_{n}^{\otimes r}+B_{n}\left(\mathcal{U}^{l}\right) D^{r} U_{n}^{l}+R_{n, r}\left(\mathcal{U}^{l}\right) .
$$

That is, denoting $D^{r} U_{n}^{l}$ by $T_{n}^{l}, \mathcal{T}^{l}=\left\{T_{n}^{l}\right\}_{n \in \mathbb{Z}}$, we have that

$$
\mathcal{T}^{l+1}=\mathcal{A}\left(\mathcal{U}^{l}\right) \mathcal{T}^{l}+\mathcal{R}\left(\mathcal{U}^{l}\right),
$$

with

$$
\begin{aligned}
\left(\mathcal{A}\left(\mathcal{U}^{l}\right) \mathcal{T}^{l}\right)_{n} & =\left(A_{n}^{S^{c}}\right)^{-1} T_{n+1}^{l} \circ \psi\left(\mathcal{U}^{l}\right)_{n} D \psi\left(\mathcal{U}^{l}\right)_{n}^{\otimes r}+B_{n}\left(\mathcal{U}^{l}\right) T_{n}^{l}, \\
\mathcal{R}\left(\mathcal{U}^{l}\right)_{n} & =R_{n, r}\left(\mathcal{U}^{l}\right) .
\end{aligned}
$$

We have that $\mathcal{A}\left(\mathcal{U}^{l}\right)$ is a linear map from

$$
\begin{array}{r}
\Xi=\left\{\mathcal{T}=\left\{T_{n}\right\}_{n \in \mathbb{Z}}: T_{n} \in C^{0}\left(B(0,1), L^{r}\left(\mathbb{R}^{d} ; \mathbb{R}^{d}\right)\right),\right. \\
\left.\omega\left(T_{n}, \eta\right) \text { uniformly bounded in } n\right\}
\end{array}
$$

to itself. Note that the terms of the sequence $\mathcal{T}^{l}$ belong to $\Xi$. Moreover both $\mathcal{A}(\mathcal{U})$ and $\mathcal{R}(\mathcal{U})$ are continuous in the $C^{0}$-norm when $\mathcal{U}$ is $C^{r-1}$ with modulus of continuity of the $r-1$ derivative bounded.

We claim that the sequence $\mathcal{T}^{l}$ converges in the $C^{0}$-norm to a continuous map. Indeed, this limit will be the only bounded solution, $\mathcal{T}^{\infty}$, of the equation

$$
\mathcal{T}=\mathcal{A}\left(\mathcal{U}^{\infty}\right) \mathcal{T}+\mathcal{R}\left(\mathcal{U}^{\infty}\right)
$$

This equation has a unique solution since

$$
\left\|\mathcal{A}\left(\mathcal{U}^{\infty}\right)\right\| \leq \sup _{n \in \mathbb{Z}}\left\|\left(A_{n}^{S^{c}}\right)^{-1}\right\|\left(\left\|A_{n}^{S}\right\|+\nu\right)^{r}+\nu<\gamma<1,
$$

and, hence, the right-hand side of (46) is a contraction. We can also assume that $\left\|\mathcal{A}\left(\mathcal{U}^{l}\right)\right\| \leq \gamma$. To prove the claim we check that $\mathcal{T}^{l} \rightarrow \mathcal{T}^{\infty}$ in the $C^{0}$-norm when $l \rightarrow \infty$,

$$
\begin{aligned}
\left\|\mathcal{T}^{l}-\mathcal{T}^{\infty}\right\| & \leq\left\|\mathcal{A}\left(\mathcal{U}^{l-1}\right) \mathcal{T}^{l-1}-\mathcal{A}\left(\mathcal{U}^{\infty}\right) \mathcal{T}^{\infty}\right\|+\left\|\mathcal{R}\left(\mathcal{U}^{l-1}\right)-\mathcal{R}\left(\mathcal{U}^{\infty}\right)\right\| \\
& \leq \gamma\left\|\mathcal{T}^{l-1}-\mathcal{T}^{\infty}\right\|+d_{l}
\end{aligned}
$$


where

$$
d_{l}=\left\|\left(\mathcal{A}\left(\mathcal{U}^{l-1}\right)-\mathcal{A}\left(\mathcal{U}^{\infty}\right)\right) \mathcal{T}^{\infty}\right\|+\left\|\mathcal{R}\left(\mathcal{U}^{l-1}\right)-\mathcal{R}\left(\mathcal{U}^{\infty}\right)\right\|
$$

Note that by the continuity of $\mathcal{A}$ and $\mathcal{R}, d_{l} \rightarrow 0$ when $l \rightarrow \infty$. Then it is clear that

$$
\left\|\mathcal{T}^{l}-\mathcal{T}^{\infty}\right\| \leq \gamma^{l-1}\left\|\mathcal{T}^{1}-\mathcal{T}^{\infty}\right\|+\sum_{j=0}^{l-1} \gamma^{j} d_{l-j}
$$

which tends to 0 when $l \rightarrow \infty$ since $\gamma<1$ and $d_{l} \rightarrow 0$.

This proves that $D^{r} U_{n}^{l}$ tends in the $C^{0}$-norm to $T_{n}^{\infty}$. To check that this map is the $r$ derivative of $\mathcal{U}^{\infty}$ we simply note that for all $n$

$$
D^{r-1} U_{n}^{l}(y)-D^{r-1} U_{n}^{l}(x)=\int_{0}^{1} D^{r} U_{n}^{l}(x+s(y-x))(y-x) d s .
$$

Since the integrand in the right-hand side converges uniformly to the continuous $\operatorname{map} T_{n}^{\infty}$, we have

$$
\begin{aligned}
D^{r-1} U_{n}^{\infty}(y)-D^{r-1} U_{n}^{\infty}(x)= & T_{n}^{\infty}(x)(y-x) \\
& +\int_{0}^{1}\left[T_{n}^{\infty}(x+s(y-x))-T_{n}^{\infty}(x)\right](y-x) d s
\end{aligned}
$$

and hence $D^{r} U_{n}^{\infty}(x)=T_{n}^{\infty}(x)$.

To prove the case when $r=\infty$, we note that when $f_{n} \in C^{\infty}$, we can find a sequence of positive numbers $\rho_{N_{S}+2}, \ldots, \rho_{k}$ such that $\sigma\left(\mathcal{B}\left(\rho_{N_{S}+2}, \ldots, \rho_{k}\right)\right) \subset$ $\mathcal{B}\left(\rho_{N_{S}+2}, \ldots, \rho_{k}\right)$, for all $k \geq N_{S}+2$. According to the preceding arguments the fixed point of $\sigma$ is $C^{r-1+\text { lip }}$ for all $k$, and hence $C^{\infty}$.

The case $r=\omega$ is much easier. It just suffices to observe that the previous arguments work exactly in the same way in a complex ball. We consider the Banach space of functions, analytic in the open ball, continuous on the closed ball, and vanishing to order $N_{S}$ at the origin topologized with the supremum of the $N_{S}+1$ derivative.

We have established the existence claim of Theorem 2.5, (a) and (b). To prove the uniqueness statement (e) consider $\mathcal{V}=\left\{V_{n}\right\}_{n \in \mathbb{Z}}, \hat{\mathcal{V}}=\left\{\hat{V}_{n}\right\}_{n \in \mathbb{Z}}$. From (b), $\mathcal{V}=\sigma(\mathcal{V})$ and $\hat{\mathcal{V}}=\sigma(\hat{\mathcal{V}})$. Then

$$
\|\mathcal{V}-\hat{\mathcal{V}}\|_{\mathcal{X}^{0}}=\|\sigma(\mathcal{V})-\sigma(\hat{\mathcal{V}})\|_{\mathcal{X}^{0}} \leq \operatorname{Lip} \sigma_{\mid \mathcal{B}_{N_{S}+1}}\|\mathcal{V}-\hat{\mathcal{V}}\|_{\mathcal{X}^{0}}
$$

and $\operatorname{Lip} \sigma_{\mid \mathcal{B}_{N_{S}+1}}<1$ in the $\mathcal{X}^{0}$-norm. This shows that $\mathcal{V}=\hat{\mathcal{V}}$

To prove statement (f), we observe that (16) shows that if $\hat{V}_{n}$ is $C^{k}, k \leq r$, for some $n$, then it is $C^{k}$ for all $n$. Then, we just have to obtain uniform estimates for the derivatives, assuming they exist. We shall consider the case $k=N_{S}+1$. The other cases follow by induction.

If we take $N_{S}+1$ derivatives of (16), we obtain in a similar way as for (32)

$$
\begin{aligned}
D^{N_{S}+1} \hat{V}_{n}(x)= & \left(A_{n}^{S^{c}}\right)^{-1} D^{N_{S}+1} \hat{V}_{n+1}\left(A_{n}^{S} x+\Pi^{S} \tilde{f}_{n}\left(x, \hat{V}_{n}(x)\right)\right)\left(A_{n}^{S}\right)^{\otimes\left(N_{S}+1\right)} \\
& +Q_{n, N_{S}+1}(\hat{\mathcal{V}}),
\end{aligned}
$$

where $Q_{n, N_{S}+1}(\hat{\mathcal{V}})$ contains terms with derivatives up to order $N_{S}+1$ of $\hat{V}_{n}$ and $\hat{V}_{n+1}$, but all of them multiplied by factors which involve derivatives of $\tilde{f}_{n}$ up to order $N_{S}+1$. 
From this observation, we conclude that

$$
\begin{aligned}
\left\|D^{N_{S}+1} \hat{V}_{n}\right\|_{C^{0}} \leq & \left\|\left(A_{n}^{S^{c}}\right)^{-1}\right\|\left\|A_{n}^{S}\right\|^{N_{S}+1}\left\|D^{N_{S}+1} \hat{V}_{n+1}\right\|_{C^{0}} \\
& +\nu\left(\left\|\hat{V}_{n}\right\|_{C^{N_{S}+1}}+\left\|\hat{V}_{n+1}\right\|_{C^{N_{S}+1}}\right),
\end{aligned}
$$

where $\nu$ can be made as small as we need by assuming that $\|\tilde{\mathcal{F}}\|_{C^{r}}$ is sufficiently small.

We observe that

$$
\begin{aligned}
\left\|\hat{V}_{n}\right\|_{C^{N_{S}+1}} & \leq \sup \left(\left\|\hat{V}_{n}\right\|_{C^{N_{S}}},\left\|D^{N_{S}+1} \hat{V}_{n}\right\|_{C^{0}}\right) \\
& \leq\|\hat{\mathcal{V}}\|_{C^{N_{S}}}+\left\|D^{N_{S}+1} \hat{V}_{n}\right\|_{C^{0}} .
\end{aligned}
$$

Substituting (49) into (48) and using the fact that $\left\|\left(A_{n}^{S^{c}}\right)^{-1}\right\|\left\|A_{n}^{S}\right\|^{N_{S}+1}<1$, we obtain that

$$
\left\|D^{N_{S}+1} \hat{V}_{n}\right\|_{C^{0}} \leq \gamma\left\|D^{N_{S}+1} \hat{V}_{n+1}\right\|_{C^{0}}+D
$$

where $\gamma<1$ and $D$ is some constant independent of $n$. Statement (f) now follows easily.

\section{An eXAmple}

The following example illustrates some of the subtle phenomena involved in slow manifolds showing that uniqueness may or may not hold depending very much on the details of the conditions. In particular, it shows that some of the limitations in Theorem 2.5 do belong.

This example is first presented as a family of maps, as in the setting of Theorem 2.5 and then, at the end of this section, we will show that this family can be lifted to a smooth map from a four-dimensional compact manifold to itself. The construction of such a lift is explicit and is quite similar to the construction in Pug84.

Example 3.1. Consider the sequence of maps $f_{n}: \mathbb{R}^{2} \rightarrow \mathbb{R}^{2}, n \in \mathbb{Z}$, defined by

$$
\begin{aligned}
& f_{n}\left(x_{1}, x_{2}\right)=\left(\frac{1}{3} x_{1}, \frac{1}{20} x_{2}\right), \quad n \neq 0, \\
& f_{0}\left(x_{1}, x_{2}\right)=\left(\frac{1}{3} x_{1}, \frac{1}{20} x_{2}+\varphi\left(x_{1}, x_{2}\right)\right),
\end{aligned}
$$

where $\varphi$ is a $C^{\infty}$ real-valued function with compact support — which we will think of as very small — not including $(0,0)$ and

$$
\sup _{\left(x_{1}, x_{2}\right) \in \mathbb{R}^{2}}\left|\frac{\partial \varphi}{\partial x_{2}}\left(x_{1}, x_{2}\right)\right|<\frac{1}{20} .
$$

Clearly, we have

$$
D f_{n}(0,0)=\left(\begin{array}{cc}
\frac{1}{3} & 0 \\
0 & \frac{1}{20}
\end{array}\right), \quad n \in \mathbb{Z} .
$$

Moreover, condition (51) ensures that each $f_{n}, n \in \mathbb{Z}$, is a bijective map. Indeed, this assertion is trivial for $n \neq 0$. For $n=0$, we remark that, given $\left(z_{1}, z_{2}\right) \in \mathbb{R}^{2}$, the equation

is equivalent to

$$
f_{0}\left(x_{1}, x_{2}\right)=\left(z_{1}, z_{2}\right)
$$

$$
x_{1}=3 z_{1}, \quad x_{2}=20 z_{2}-20 \varphi\left(3 z_{1}, x_{2}\right) .
$$


The second condition has a unique solution, since its right-hand side defines a contraction on $\mathbb{R}$. Hence, since $\operatorname{det} D f_{n}\left(x_{1}, x_{2}\right) \neq 0$, for all $\left(x_{1}, x_{2}\right) \in \mathbb{R}^{2}$, the functions $f_{n}, n \in \mathbb{Z}$, are global diffeomorphisms.

The sequence of maps (50) satisfies Definition 1.2 if we take as $E_{n}^{i}$ the coordinate axes and we set $\lambda_{1}=\mu_{1}=\log (1 / 3), \lambda_{2}=\mu_{2}=\log (1 / 20), \ell=1, \varepsilon=0$. We take $E_{n}^{S}$ to consist of just the first coordinate axis. In such a case, $N_{S}=[\log 20 / \log 3]=2$.

The set of manifolds graph $\left(V_{n}\right)$ satisfies the condition

$$
f_{n}\left(\operatorname{graph}\left(V_{n}\right)\right)=\operatorname{graph}\left(V_{n+1}\right) \quad \text { for all } n \in \mathbb{Z}
$$

if and and only if the functions $V_{n}$ satisfy

$$
V_{n+1}\left(x_{1}\right)=\frac{1}{20} V_{n}\left(3 x_{1}\right)+\delta_{n, 0} \varphi\left(3 x_{1}, V_{n}\left(3 x_{1}\right)\right)
$$

where $\delta$ is the $\delta$ of Kronecker.

Furthermore, it is easy to verify by induction in $n$ that a sequence of functions $V_{n}$ satisfying (52) also satisfies the initial condition

$$
V_{0}=\Psi
$$

where $\Psi: \mathbb{R} \rightarrow \mathbb{R}$ is a $C^{\infty}$ function with compact support such that $\Psi(0)=0$, if and only if it is of the form

$$
V_{n}\left(x_{1}\right)= \begin{cases}\left(\frac{1}{20}\right)^{n}(\Psi+20 \varphi \circ(\mathrm{Id}, \Psi))\left(3^{n} x_{1}\right), & n \geq 1 \\ \left(\frac{1}{20}\right)^{n} \Psi\left(3^{n} x_{1}\right), & n \leq 0 .\end{cases}
$$

We see that

$$
\frac{d^{j}}{d x_{1}^{j}} V_{n}\left(x_{1}\right)= \begin{cases}\left(\frac{3^{j}}{20}\right)^{n} \frac{d^{j}}{d x_{1}^{j}}(\Psi+20 \varphi \circ(\mathrm{Id}, \Psi))\left(3^{n} x_{1}\right), & n \geq 1 \\ \left(\frac{3^{j}}{20}\right)^{n} \frac{d^{j}}{d x_{1}^{j}}(\Psi)\left(3^{n} x_{1}\right), & n \leq 0\end{cases}
$$

We make the following observations:

(i) Since $V_{n}^{\prime}(0)=\left(\frac{3}{20}\right)^{n} \Psi^{\prime}(0)$ we see that the derivative is unbounded unless $\Psi^{\prime}(0)=0$. In such a case, $V_{n}^{\prime}(0)=0$ for all $n \in \mathbb{Z}$. This phenomenon of boundedness of first derivatives implying tangency is an illustration of part (d) of Theorem 1.9 .

(ii) Suppose that $\Psi$ has support not containing 0 . If $j \leq N_{S}$, equivalently, $3^{j} / 20<1$, we have that $\left|V_{n}^{(j)}\left(x_{1}\right)\right|$ is bounded uniformly on $n$ in a ball around the origin. This follows by observing that for $n>0$, we have uniform boundedness in (54) because of the factor $\left(\frac{3^{j}}{20}\right)^{n}$. For $n<0$, we have boundedness because for $n$ sufficiently negative, the support of $V_{n}$ is outside of the unit ball. This illustrates that we cannot expect uniqueness by only assuming boundedness of derivatives of order less than $N_{S}$.

(iii) Let $j>N_{S}$. Choose $\varphi \equiv 0$ and $\Psi$ such that $\Psi(0)=0, \Psi^{\prime}(0)=0$ and $\Psi^{(j)}(0) \neq 0$. Then, it is easy to see that $\left|V_{n}\right|_{C^{j}}$ is bounded for $n$ negative but not for $n$ positive.

(iv) If $j>N_{S}$, the only possibility of having uniform bounds for $\left|V_{n}^{(j)}\left(x_{1}\right)\right|$ when $n>0$ is that

$$
\Psi=-20 \varphi \circ(\mathrm{Id}, \Psi)
$$


We claim that this equation has a unique continuous solution which is $C^{\infty}$ and has compact support. Indeed, for any $x \in \mathbb{R}$, condition (51) implies that the right-hand side of the equation

$$
y=-20 \varphi(x, y)
$$

is a contraction, and, hence, has a unique solution $\Psi(x)$. Since $\varphi$ has compact support, $\Psi(x)$ also has compact support. The standard implicit function theorem ensures that $\Psi$ is $C^{\infty}$. This uniquely determines the functions $V_{n}$ for all values of $n$. Note that for these functions $\left|V_{n}^{(j)}\left(x_{1}\right)\right|$ is uniformly bounded for $n<0$.

This illustrates the fact that we have uniqueness under the assumption of uniform boundedness of the derivatives of order bigger than $N_{S}$.

Now we show how to embed this family in a smooth map. In the following, we will denote by $\mathbb{S}^{2}$ the two-dimensional sphere.

Proposition 3.2. Consider the family of maps $\left\{f_{n}\right\}_{n \in \mathbb{Z}}$ of Example 3.1. Then there exists a two-dimensional compact smooth manifold $M^{2}$, a smooth map $F$ : $\mathbb{S}^{2} \times M^{2} \rightarrow \mathbb{S}^{2} \times M^{2}$, a point $z_{0} \in \mathbb{S}^{2} \times M^{2}$ with orbit $\left\{z_{n}=F^{n}\left(z_{0}\right)\right\}$, and smooth two-dimensional submanifolds $N_{n} \subset \mathbb{S}^{2} \times M^{2}$ such that

i) $z_{n} \in N_{n}, F\left(N_{n}\right) \subset N_{n+1}$.

ii) There exists a diffeomorphism $\sigma$ such that $F_{\mid N_{n}}=\sigma^{-1} \circ f_{n} \circ \sigma, n \in \mathbb{Z}$.

Proof. The construction of the map $F$ is performed in two steps. The first one consists of lifting the discrete family $\left\{f_{n}\right\}$ to a smooth family of maps $\left\{\tilde{g}_{\lambda}\right\}_{\lambda \in[0,1]}$ with $\tilde{g}_{\lambda}: \mathbb{S}^{2} \rightarrow \mathbb{S}^{2}$. In the second step, with the aid of an auxiliary map on a compact smooth manifold, exhibiting sufficiently rich hyperbolic dynamics - a Smale horseshoe, for instance - the map $F$ and the orbit are explicitly given.

First we introduce some notation. We fix $\mathbb{S}^{2}$ to be $\left\{(x, y, z) \in \mathbb{R}^{3}: x^{2}+y^{2}+z^{2}=\right.$ $1\}, \mathbb{S}_{+}^{2}=\mathbb{S}^{2} \cap\{z>0\}, \mathbb{S}_{-}^{2}=\mathbb{S}^{2} \cap\{z<0\}$ and $E=\mathbb{S}^{2} \cap\{z=0\}$. Let $\pi: \mathbb{S}_{-}^{2} \rightarrow \mathbb{R}^{2}$ be the map

$$
\pi(x, y, z)=\left(-\frac{x}{z},-\frac{y}{z}\right) .
$$

Note that $\pi$ is the projection of $\mathbb{S}^{2}$ from the center of the sphere onto the plane $\{z=-1\}$. We remark that $\pi$ is a diffeomorphism. We denote $\pi^{-1}$ by $\sigma$. It is clear that

$$
\sigma\left(x_{1}, x_{2}\right)=\left(\frac{x_{1}}{\sqrt{1+x_{1}^{2}+x_{2}^{2}}}, \frac{x_{2}}{\sqrt{1+x_{1}^{2}+x_{2}^{2}}}, \frac{-1}{\sqrt{1+x_{1}^{2}+x_{2}^{2}}}\right) .
$$

We also consider the antipodal map $\mu: \mathbb{S}^{2} \rightarrow \mathbb{S}^{2}$, that is, $\mu(p)=-p$. In this way, $\sigma$ is a chart of $\mathbb{S}^{2}$ covering $\mathbb{S}_{-}^{2}$ and $\mu \circ \sigma$ is a chart covering $\mathbb{S}_{+}^{2}$.

We define the one-parameter family of maps $g_{\lambda}: \mathbb{R}^{2} \rightarrow \mathbb{R}^{2}$ by

$$
g_{\lambda}\left(x_{1}, x_{2}\right)=\left(a x_{1}, b x_{2}+\lambda \varphi\left(x_{1}, x_{2}\right)\right),
$$

where $a=1 / 3$ and $b=1 / 20$. We have that $g_{1}=f_{0}$ and $g_{0}=f_{n}, n \in \mathbb{Z} \backslash\{0\}$. Now we define a lift of $g_{\lambda}$ to $\mathbb{S}_{-}^{2}$ by

$$
\tilde{g}_{\lambda}^{-}=\sigma \circ g_{\lambda} \circ \pi
$$

a lift to $\mathbb{S}_{+}^{2}$ by

$$
\tilde{g}_{\lambda}^{+}=\mu \circ \tilde{g}_{\lambda}^{-} \circ \mu^{-1}
$$


and finally

$$
\tilde{g}_{\lambda}(p)= \begin{cases}\tilde{g}_{\lambda}^{+}(p), & p \in \mathbb{S}_{+}^{2}, \\ \tilde{g}_{\lambda}^{-}(p), & p \in \mathbb{S}_{-}^{2} .\end{cases}
$$

From (56) and (57) we have that, for $(x, y, z) \in \mathbb{S}^{2}, z \neq 0$,

$$
\tilde{g}_{\lambda}(x, y, z)=\left(\frac{a x}{\rho(x, y, z)}, \frac{b y-\lambda z \varphi(-x / z,-y / z)}{\rho(x, y, z)}, \frac{z}{\rho(x, y, z)}\right),
$$

where

$$
\rho(x, y, z)=\sqrt{z^{2}+a^{2} x^{2}+(b y-\lambda z \varphi(-x / z,-y / z))^{2}} .
$$

We remark that $\rho$ never vanishes. Since $\varphi$ has compact support, $\tilde{g}_{\lambda}$ extends to a $C^{\infty}$ map defined in the whole sphere. Indeed, if we take $R=\inf _{r \in \mathbb{R}}\{r: \operatorname{supp}(\varphi) \subset$ $\left.D_{r}(0)\right\}$, we have that $\varphi(u, v)=0$, for $|(u, v)|>R$. Then, for any $(x, y, z) \in \mathbb{S}^{2}$ such that $|z|<1 / \sqrt{1+R^{2}}$, we take $\rho(x, y, z)=\sqrt{z^{2}+a^{2} x^{2}+b^{2} y^{2}}$ and

$$
\tilde{g}_{\lambda}(x, y, z)=\left(\frac{a x}{\rho(x, y, z)}, \frac{b y}{\rho(x, y, z)}, \frac{z}{\rho(x, y, z)}\right),
$$

which is $C^{\infty}$ in a neighborhood of $E$ and extends both $\tilde{g}_{\lambda \mid \mathbb{S}_{+}^{2}}$ and $\tilde{g}_{\lambda \mid \mathbb{S}_{-}^{2}}$ to $\mathbb{S}^{2}$. Therefore, for each $\lambda \in[0,1], \tilde{g}_{\lambda}: \mathbb{S}^{2} \rightarrow \mathbb{S}^{2}$ is a diffeomorphism, which preserves $\mathbb{S}_{-}^{2}, \mathbb{S}_{+}^{2}$ and $E$, and that, restricted to $\mathbb{S}_{-}^{2}, \tilde{g}_{\lambda}$ is conjugated to $g_{\lambda}$ through $\pi$ and $\sigma$. Moreover, the dependence on the parameter $\lambda$ is smooth.

Now, we consider any compact two-dimensional smooth manifold $M^{2}$, and a diffeomorphism $h: M^{2} \rightarrow M^{2}$ with an invariant hyperbolic subset $\Sigma$ such that $h_{\mid \Sigma}$ is conjugated to the Bernoulli shift with two symbols. We can, for example, take $h$ to be a Smale horseshoe. We can also assume that the Lyapunov exponents of this hyperbolic set are bigger than $-\log 3$, in order to avoid resonances. Let $q_{0} \in \Sigma$ be the point corresponding to the sequence $(\cdots 11011 \cdots)$. Clearly, there exists a $C^{\infty}$ function with compact support, $\eta: M^{2} \rightarrow[0,1]$, such that $\eta \equiv 1$ in a neighborhood of $q_{0}$ and vanishes outside a compact set which does not include $h^{n}\left(q_{0}\right), n \neq 0$, that is, $\eta\left(h^{n}\left(q_{0}\right)\right)=\delta_{n, 0}, n \in \mathbb{Z}$. We define $F: \mathbb{S}^{2} \times M^{2} \rightarrow \mathbb{S}^{2} \times M^{2}$ by

$$
F(p, q)=\left(\tilde{g}_{\eta(q)}(p), h(q)\right) .
$$

Clearly $F$ satisfies the properties listed in Proposition 3.2. We consider the orbit of the point $z_{0}=\left(\sigma(0,0), q_{0}\right)$. It is clear that $z_{n}=F^{n}\left(z_{0}\right)=\left(\sigma(0,0), q_{n}\right)$, where $q_{n}=h^{n}\left(q_{0}\right)$. By definition, the submanifolds $N_{n}=\mathbb{S}^{2} \times\left\{q_{n}\right\} \cong \mathbb{S}^{2}$ verify that $F\left(N_{n}\right)=N_{n+1}$ and $F_{\mid N_{n}}=\tilde{g}_{\eta\left(q_{n}\right)}$. Finally, $\tilde{g}_{\eta\left(q_{n}\right)}$ is conjugated to $f_{n}$ by $\sigma$, which establishes the claim.

\section{ACKNOWLEDGMENTS}

The first and third authors acknowledge the partial support of Spanish MCYT Grant BFM2003-09504-C02-01 and the Catalan Grant CIRIT 2001SGR-70. The second author has been partially supported by NSF and acknowledges the hospitality of UPC and UB as well as ICREA.

\section{REFERENCES}

[CFdlL03a] Xavier Cabré, Ernest Fontich, and Rafael de la Llave, The parameterization method for invariant manifolds I: manifolds associated to non-resonant subspaces, Indiana Univ. Math. J. 52 (2003), 283-328. MR1976079 (2004h:37030) 
[CFdlL03b] — The parameterization method for invariant manifolds II: regularity with respect to parameters, Indiana Univ. Math. J. 52 (2003), 329-360. MR.1976080 (2004h:37031)

[dlL92] R. de la Llave, Smooth conjugacy and $S-R-B$ measures for uniformly and nonuniformly hyperbolic systems, Comm. Math. Phys. 150 (1992), no. 2, 289-320. MR:1194019 (94a:58153)

[dlL97] Rafael de la Llave, Invariant manifolds associated to nonresonant spectral subspaces, J. Statist. Phys. 87 (1997), no. 1-2, 211-249. MR1453740 (98f:58038)

[dlL03] R. de la Llave, Invariant manifolds associated to invariant subspaces without invariant complements: a graph transform approach, Math. Phys. Electron. J. 9 (2003), Paper 3, 35 pp. (electronic). MR2028331(2005c:37045)

[dlLMM86] Rafael de la Llave, Jose Manuel Marco, and Roberto Moriyón, Canonical perturbation theory of Anosov systems and regularity results for the Livšic cohomology equation, Ann. of Math. (2) 123 (1986), no. 3, 537-611. MR0840722 (88h:58091)

[dlLW95] Rafael de la Llave and C. Eugene Wayne, On Irwin's proof of the pseudostable manifold theorem, Math. Z. 219 (1995), no. 2, 301-321. MR1337223 (96g:58102)

[ElB01] Mohamed S. ElBialy, Sub-stable and weak-stable manifolds associated with finitely non-resonant spectral subspaces, Math. Z. 236 (2001), no. 4, 717-777. MR1827502 (2002i:58007)

[Fen77] N. Fenichel, Asymptotic stability with rate conditions. II, Indiana Univ. Math. J. 26 (1977), no. 1, 81-93. MR0426056 (54:14002)

[Fen74] _ Asymptotic stability with rate conditions, Indiana Univ. Math. J. 23 (1973/74), 1109-1137. MR0339276 (49:4036)

[Fra88] Simon J. Fraser, The steady state and equilibrium approximations: A geometrical picture, Jour. Chem. Phys. 88 (1988), no. 8, 4732-4738.

[GK98] M. Guysinsky and A. Katok, Normal forms and invariant geometric structures for dynamical systems with invariant contracting foliations, Math. Res. Lett. 5 (1998), no. 1-2, 149-163. MR1618331 (99b:58215)

[HP70] M.W. Hirsch and C.C. Pugh, Stable manifolds and hyperbolic sets, Global Analysis (Proc. Sympos. Pure Math., Vol. XIV, Berkeley, Calif., 1968), Amer. Math. Soc., Providence, R.I., 1970, pp. 133-163. MR0271991(42:6872)

[HPS77] M.W. Hirsch, C.C. Pugh, and M. Shub, Invariant manifolds, Springer-Verlag, Berlin, 1977, Lecture Notes in Mathematics, Vol. 583. MR0501173 (58:18595)

[JPdlL95] M. Jiang, Ya. B. Pesin, and R. de la Llave, On the integrability of intermediate distributions for Anosov diffeomorphisms, Ergodic Theory Dynam. Systems 15 (1995), no. 2, 317-331. MR1332406 (96b:58087)

[LI73] Oscar E. Lanford III, Bifurcation of periodic solutions into invariant tori: the work of Ruelle and Takens, Nonlinear problems in the Physical Sciences and Biology: Proceedings of a Battelle Summer Institute (Berlin) (Ivar Stakgold, Daniel D. Joseph, and David H. Sattinger, eds.), Springer-Verlag, 1973, Lecture Notes in Mathematics, Vol. 322, pp. 159-192. MR0371548 (51:7766)

[LL04] Wei Li and Kening Lu, Sternberg theorems for random dynamical systems, MP_ARC \#04-12.

[Mat68] John N. Mather, Characterization of Anosov diffeomorphisms, Nederl. Akad. Wetensch. Proc. Ser. A 71 = Indag. Math. 30 (1968), 479-483. MR0248879 (40:2129)

[MP92] U. Maas and S. B. Pope, Simplifying chemical kinetics: intrinsic low-dimensional manifolds in composition space, Combust. Flame (1992), 239-264.

[Ose68] V. I. Oseledec, A multiplicative ergodic theorem. Characteristic Ljapunov, exponents of dynamical systems, Trudy Moskov. Mat. Obšč 19 (1968), 179-210. MR0240280 (39:1629)

[Pes73] Ja. B. Pesin, The existence of invariant foliations for a diffeomorphism of a smooth manifold, Mat. Sb. (N.S.) 91(133) (1973), 202-210, 287. MR 0343307 (49:8049)

[Pes76] - Families of invariant manifolds that correspond to nonzero characteristic exponents, Izv. Akad. Nauk SSSR Ser. Mat. 40 (1976), no. 6, 1332-1379, 1440. MR0458490 (56:16690)

[Pes77] Characteristic Ljapunov exponents, and smooth ergodic theory, Uspehi Mat. Nauk 32 (1977), no. 4 (196), 55-112, 287. MR0466791 (57:6667) 
[Pug84] Charles C. Pugh, The $C^{1+\alpha}$ hypothesis in Pesin theory, Inst. Hautes Études Sci. Publ. Math. (1984), no. 59, 143-161. MR0743817 (85k:58047)

[Rue79] D. Ruelle, Ergodic theory of differentiable dynamical systems, Inst. Hautes Études Sci. Publ. Math. (1979), no. 50, 27-58. MR0556581 (81f:58031)

[SS74] Robert J. Sacker and George R. Sell, Existence of dichotomies and invariant splittings for linear differential systems. I, J. Differential Equations 15 (1974), 429-458. MR0341458 (49:6209)

[Yom87] Y. Yomdin, Volume growth and entropy, Israel J. Math. 57 (1987), no. 3, 285-300. MR0889979 (90g:58008)

[Yom88] , Nonautonomous linearization, Dynamical systems (College Park, MD, 1986-87), Lecture Notes in Math., vol. 1342, Springer, Berlin, 1988, pp. 718-726. MR0970581 (91h:58093)

Departament de Matemàtica Aplicada i Anàlisi, Universitat de Barcelona, Gran Via, 585, 08007 Barcelona, Spain

E-mail address: fontich@mat.ub.es

Department of Mathematics, The University of Texas at Austin, Austin, Texas 78712 1082

E-mail address: llave@math.utexas.edu

Departament de Matemàtica Aplicada IV, Universitat Politècnica de Catalunya, EdC3, Jordi Girona, 1-3, 08034 Barcelona, Spain

E-mail address: martin@ma4.upc.edu 